\title{
SYK inhibitor entospletinib prevents ocular and skin GVHD in mice
}

Jonathan C. Poe, ${ }^{1}$ Wei Jia, ${ }^{1}$ Julie A. Di Paolo, ${ }^{2}$ Nancy J. Reyes, ${ }^{3}$ Ji Yun Kim, ${ }^{2}$ Hsuan Su, ${ }^{1}$ John S. Sundy, ${ }^{4}$ Adela R. Cardones, ${ }^{5}$ Victor L. Perez, ${ }^{3}$ Benny J. Chen, ${ }^{1}$ Nelson J. Chao, ${ }^{1}$ Diana M. Cardona, ${ }^{6}$ Daniel R. Saban, ${ }^{3}$ and Stefanie Sarantopoulos ${ }^{1}$

'Department of Medicine, Division of Hematologic Malignancies and Cellular Therapy, Duke University Medical Center, Durham, North Carolina, USA. ${ }^{2}$ Department of Biology, Gilead Sciences, Foster City, California, USA. ${ }^{3}$ Department of Ophthalmology, Duke University Medical Center, Durham, North Carolina, USA. ${ }^{4}$ Inflammation/Respiratory Section, Cilead Sciences, Foster City, California, USA. ${ }^{5}$ Department of Dermatology and ${ }^{6}$ Department of Pathology, Duke University Medical Center, Durham, North Carolina, USA.

Graft-versus-host disease (GVHD) is a major complication of hematopoietic stem cell transplantation (HCT). The tyrosine kinase SYK contributes to both acute and chronic CVHD development, making it an attractive target for GVHD prevention. Entospletinib (ENTO) is a second-generation highly selective SYK inhibitor with a high safety profile. Potential utility of ENTO as CVHD prophylaxis in patients was examined using a preclinical mouse model of eye and skin CVHD and ENTO-compounded chow. We found that early SYK inhibition improved blood immune cell reconstitution in GVHD mice and prolonged survival, with $60 \%$ of mice surviving to day +120 compared with $10 \%$ of mice treated with placebo. Compared with mice receiving placebo, mice receiving ENTO had dramatic improvements in clinical eye scores, alopecia scores, and skin scores. Infiltrating $\mathrm{SYK}^{+}$cells expressing B220 or F4/80, resembling SYK+ cells found in lichenoid skin lesions of chronic CVHD patients, were abundant in the skin of placebo mice but were rare in ENTOtreated mice. Thus, ENTO given early after HCT safely prevented CVHD.

Conflict of interest: JADP, JYK, and JSS are employees of, and equity holders in, Gilead Sciences.

Submitted: May 23, 2018 Accepted: August 29, 2018 Published: October 4, 2018

Reference information: JCI Insight. 2018;3(19):e122430. https://doi.org/10.1172/jici. insight.122430.

\section{Introduction}

Graft-versus-host disease (GVHD) is a common, debilitating, and frequently lethal complication in patients receiving allogeneic hematopoietic stem cell transplantation (HCT). GVHD manifests clinically in both acute $(1,2)$ and chronic (3-6) forms (aGVHD and cGVHD, respectively). While cGVHD development is reliant on inciting events early after $\mathrm{HCT}$, distinct pathobiology and clinical manifestations evolve in the late period after $\operatorname{HCT}(1,2,5,7-12)$. Skin, eye, and mouth manifestations are common at the onset of cGVHD and are often controllable with corticosteroid-based immunosuppression $(4,13)$. In cases in which cGVHD becomes refractory to first-line corticosteroid-containing regimens, some of the most devastating disease manifestations include skin sclerosis, severe ocular keratopathy, bronchiolitis obliterans (BO) syndrome, and fibrotic changes of the gastrointestinal tract, liver, and genitalia (3-6). Data suggest that once refractory cGVHD occurs, outcomes are poor, with a median life span of $<5$ years (14). Strikingly, the odds of responding to immunosuppressive therapies after refractory cGVHD develops are $<20 \%(14,15)$. Thus, effective therapeutic agents for GVHD prevention, especially cGVHD, are urgently needed.

The ultimate goal in HCT is to induce robust graft-versus-leukemia (GVL) effects while averting GVHD $(11,16,17)$. Rates and severity of both aGVHD and cGVHD remain unacceptably high in HCT patients, despite use of standard immunosuppressive agents. Total depletion of T cells may come with risk of cancer relapse related to decreased GVL (18). Likewise, while B cells are key players in cGVHD pathogenesis (19-21), global abrogation of B cells is associated with worsened cGVHD in patients who do not subsequently recover an adequate peripheral B cell compartment $(22,23)$. This is potentially because high levels of the antiapoptotic protein B cell-activating factor (BAFF) may drive the survival and effector function of pathogenic B cells (24-26). BAFF is known to act synergistically with the most proximal kinase in the B cell antigen receptor (BCR) signaling cascade, SYK (27). Pharmacologic blockade of SYK with the inhibitor fostamatinib and genetic blockade of SYK each reduced established lung pathology in the BO mouse model of cGVHD (28). B cells isolated from patients with active cGVHD have significantly enhanced signaling 
through SYK, along with its downstream adapter $\operatorname{BLNK}(29,30)$, and cGVHD B cells, but not T cells, were preferentially killed by fostamatinib (28). Thus, we and others have hypothesized that aberrantly activated B cells can be eradicated preferentially in CGVHD using small-molecule inhibitors of BCR signaling (31). The role of aberrant B cell signaling in the pathogenesis of cGVHD has been elucidated in recent years ( 17 , 20, 21, 24, 32), and mechanisms of B cell-mediated pathology are being actively investigated. Pathogenic alloantibody (allo $\mathrm{Ab}$ ) production against host tissues disrupts recipient thymic epithelial cells in mice (19). Importantly, BO is dependent on alloAb production and germinal center B cells for disease development $(20,28)$. Likewise, alloAbs have been recently demonstrated to exacerbate cGVHD of the skin in mice (19), and fostamatinib administration has shown efficacy in a model of sclerodermatous cGVHD (33).

Several small-molecule inhibitors of lymphocyte signaling are currently being tested in clinical trials for treatment of new onset or refractory cGVHD (https://www.clinicaltrials.gov/; NCT02337517, NCT02611063, NCT02759731, NCT02841995, NCT02959944, NCT02997280, NCT03112603, NCT03415867, NCT03474679, NCT03584516, and NCT03616184) (1, 3, 9, 34). Despite strong rationale to employ these and similar agents early after HCT, few are being examined as prophylactic agents outside of phase I trials. In part, this is because of concern regarding potential effects on immune cell recovery. In addition to B cell signaling, fostamatinib blocked $\mathrm{T}$ cell activation and signaling in a mouse model of aGVHD (35). SYK is also known to mediate signaling through high-affinity $\mathrm{Fc}$ receptors of innate immune cells, with potential implications for antibody-dependent cellular cytotoxicity mediated by macrophages, NK cells, and neutrophils, as well as basophil/mast cell degranulation $(27,36,37)$. In addition to $\mathrm{T}$ cells and $\mathrm{B}$ cells, macrophages are known to play a key role in GVHD pathogenesis, particularly cGVHD. In mouse models of cGVHD involving the skin, it was recently demonstrated that fibrosis is associated with the infiltration of the dermis by $\mathrm{F} 4 / 80^{+}$macrophages that depend on colony-stimulating factor 1 receptor (CSF-1R) activation, with the exacerbation of cutaneous fibrosis and macrophage infiltration upon treatment of mice with CSF-1 (38). Targeting these infiltrating CSF-1Rdependent macrophages with an anti-CSF-1R monoclonal antibody significantly reduced cutaneous CGVHD in mice (38), but agents to eradicate macrophages have not yet been developed in clinical HCT.

Whether administration of an agent early after HCT that blocks immune cell signaling and GVHD genesis also potentially hinders robust immune cell recovery remains unknown. Thus, we carried out a preclinical study to evaluate the efficacy of entospletinib (ENTO), a second-generation SYK inhibitor with a superior target selectivity profile over that of fostamatinib (39-41), on the clinical aspects of GVHD. Our study was feasible and informative because of (a) the availability of chow formulated with ENTO that reliably blocks in vitro SYK autophosphorylation and (b) the ability to characterize and track prominent, clinical ocular and skin manifestations of GVHD in mice. We found that, when administered before signs of disease, ENTO effectively mitigated ocular and skin GVHD and significantly improved survival, compared with placebo. Along with improved disease, ENTO-treated mice had fewer SYK ${ }^{+}$cells in the skin, and this was associated with significantly increased lymphocyte and monocyte blood counts. Our study supports the potential implementation of ENTO as part of a prophylactic arsenal that specifically targets molecules critical for GVHD pathogenesis.

\section{Results}

Orally administered ENTO provided robust SYK target pharmacodynamic coverage following HCT in mice. Female $\mathrm{BALB} / \mathrm{c}$ recipient mice were lethally irradiated $(8.5 \mathrm{~Gy})$ and transplanted i.v. with $1 \times 10^{7} \mathrm{~T}$ cell-depleted C57BL/ 6 bone marrow (BM) cells alone as controls (BM only) or with $1 \times 10^{7} \mathrm{~T}$ cell-depleted C57BL/6 BM cells plus $1 \times 10^{6}$ C57BL/6 splenocytes to induce GVHD (+Spl) (Supplemental Figure 1; supplemental material available online with this article; https://doi.org/10.1172/jci.insight.122430DS1). Starting on day +12 after HCT, recipient mice were fed only chow formulated with ENTO at a concentration of $0.06 \%$ or $0.02 \%$ or placebo chow. Seven days after ENTO or placebo chow was started (day +19 after HCT), plasma samples were obtained from different, randomly selected mice at 8-hour intervals over a 24-hour period for pharmacodynamic studies. The mean ENTO plasma concentration was approximately 3-fold higher (3.48 $\mu \mathrm{M})$ for mice receiving $0.6 \%$ drug compared with a mean of $1.33 \mu \mathrm{M}$ for mice receiving $0.02 \%$ drug (Figure $1 \mathrm{~A}$ ). An estimate of the SYK pharmacodynamic effect was made by extrapolating the plasma concentration of ENTO to that required to inhibit autophosphorylation of SYK upon in vitro treatment of mouse whole-blood samples with pervanadate, as described previously (41). The ENTO plasma concentrations shown in Figure 1A ranged

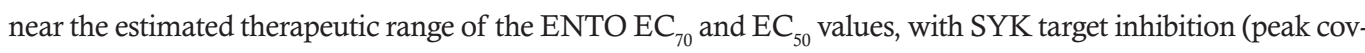
erage/average coverage) of $85 \% / 66 \%$ for the $0.06 \%$ dose and $62 \% / 32 \%$ for the $0.02 \%$ dose. Mice in all groups 

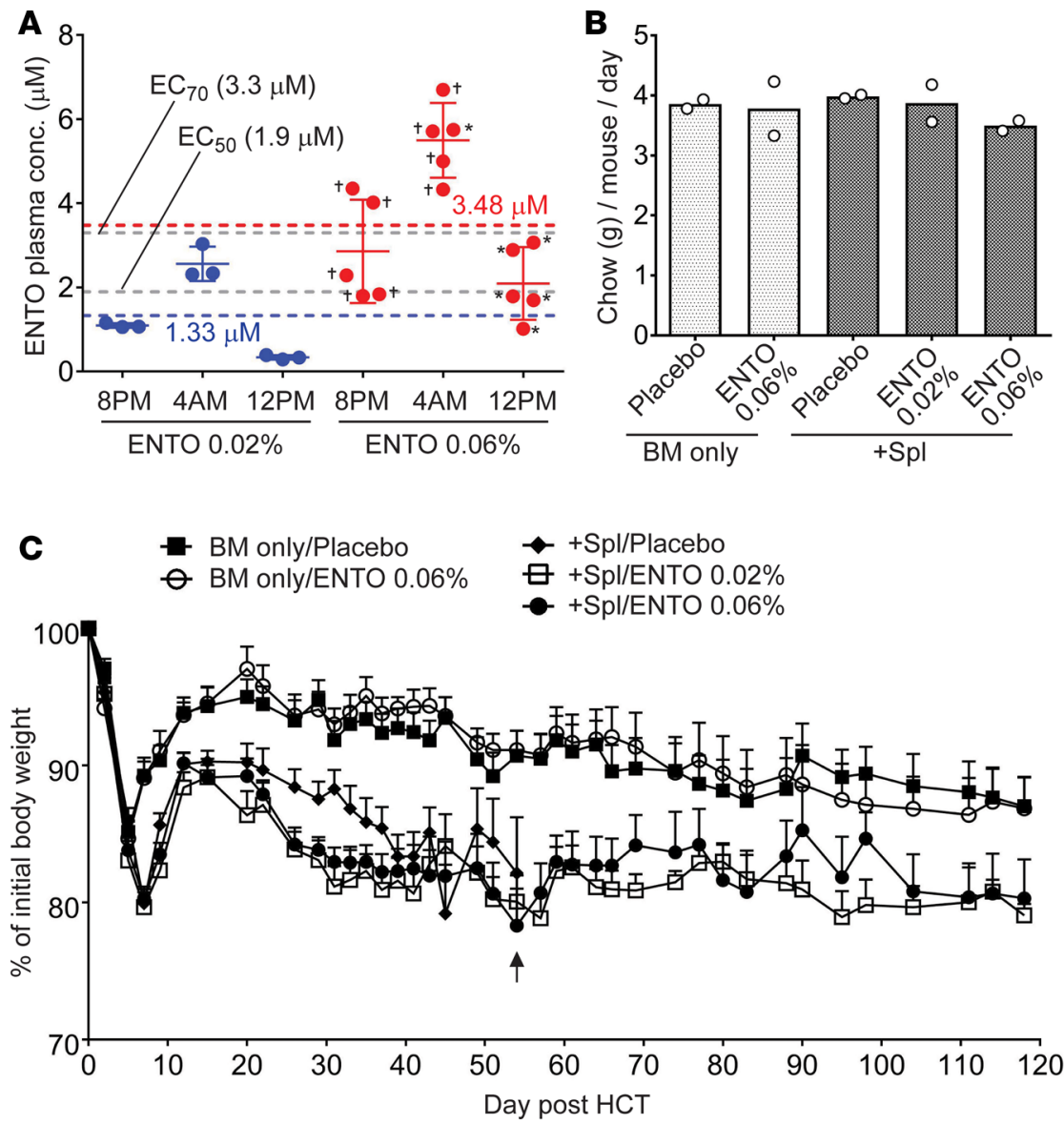

Figure 1. Orally administered ENTO reaches expected therapeutic range as assessed in pharmacokinetic studies. (A) 7 days after ENTO initiation, representative mice from HCT groups fed either $0.02 \%$ ENTO or $0.06 \%$ ENTO were bled at 8-hour intervals over a 24-hour period, and plasma was assessed for ENTO levels (performed by Agilux Laboratories Inc.). Each symbol represents an individual mouse (blue, $0.02 \%$ dose; red, $0.06 \%$ dose). Error bars represent the mean \pm SD. Average ENTO levels for each dose over the entire 24 -hour time period are indicated by the colored dashed lines $(0.02 \%$ ENTO, blue; $0.06 \%$ ENTO, red). Calculated $\mathrm{EC}_{70}$ and $\mathrm{EC}_{50}$ values derived from a standardized in vitro SYK inhibition assay (41) are as indicated by the gray dashed lines. $0.02 \%$ ENTO groups consisted of +Spl mice only. $0.06 \%$ ENTO groups consisted of both $+\mathrm{Spl}$ mice (asterisks) and BM-only mice (crosses). (B) Bars indicate the median \pm range chow consumption per mouse (grams/day) in each HCT group over the first 7 days following ENTO initiation. Each symbol represents the average values for mice housed in an individual cage, with 2 cages per group in the study. (C) All mice in each HCT group ( $n=9-10$ mice/group at day 0 ) were weighed over time on the days indicated by symbols in each graph. Values represent mean \pm SEM percentage of body weight on the day indicated compared with the weight on the day that HCT was performed (day 0). ENTO administration was initiated on day +12 . The arrow indicates the time (day +54$)$ after which only 1 mouse in the $+\mathrm{Spl} /$ placebo group remained alive, and so no further weight tracking is shown for this group.

were observed to consume their chow in normal daily amounts, as assessed during the first 7 days following ENTO/placebo initiation (Figure 1B). Notably, the approximately 15\% loss in body weight characteristic of cGVHD mouse models was evident in all $3 \mathrm{GVHD}(+\mathrm{Spl}$ ) groups (Figure 1C). Regardless, the fact that ENTO plasma levels reached the expected therapeutic range in both GVHD and BM-only control mice (Figure 1A) and chow consumption was normal in ENTO-treated GVHD mice following drug initiation (Figure 1B) indicated that ENTO uptake was not impaired in the context of reduced weight. ENTO itself had no negative effect on body mass, as ENTO-treated BM-only control mice maintained their weight at levels nearly identical to that of placebo-treated BM-only control mice (Figure 1C).

ENTO blocked development of clinical eye disease and alopecia in GVHD mice. Mice in each HCT group were followed over time for clinical signs of GVHD, including eye and skin findings. By the end of the first month after HCT, most mice in the placebo-treated GVHD (+Spl) group developed obvious clinical ocular disease and ruffled facial fur (Figure 2A and data not shown). In collaboration with investigators at the Duke University Eye Center, we employed previously validated microscopic examinations and obtained eye scores, 
A

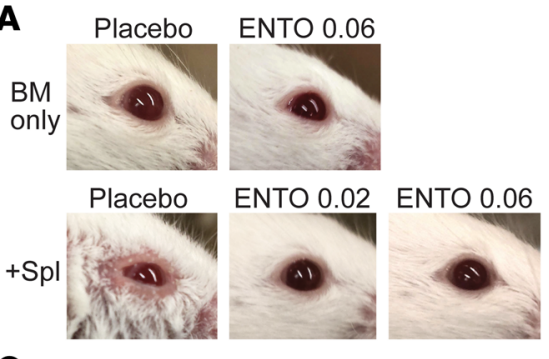

C
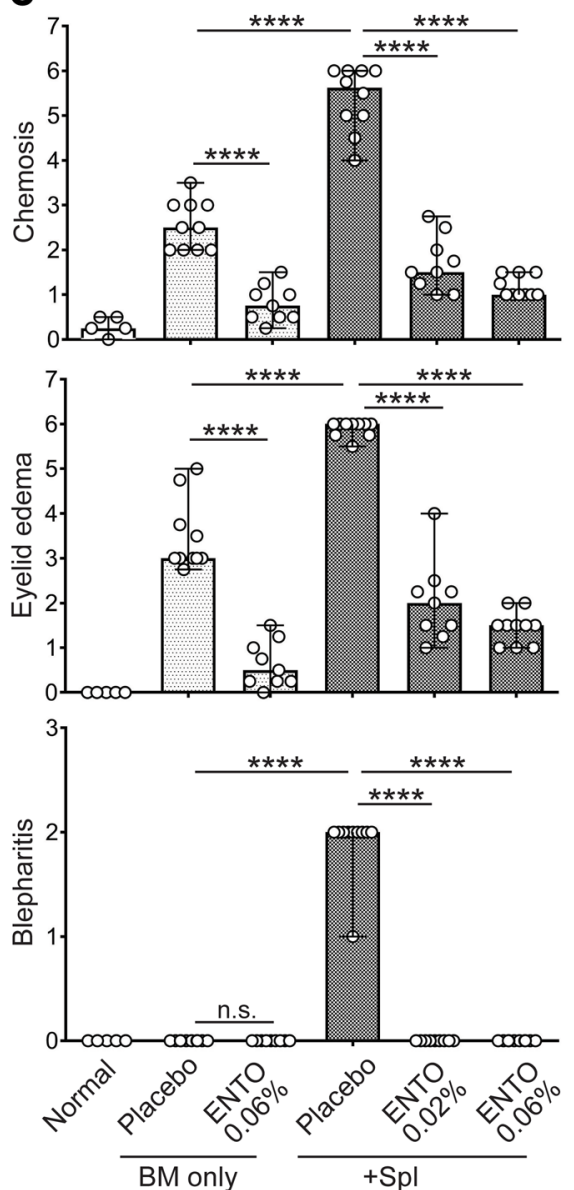
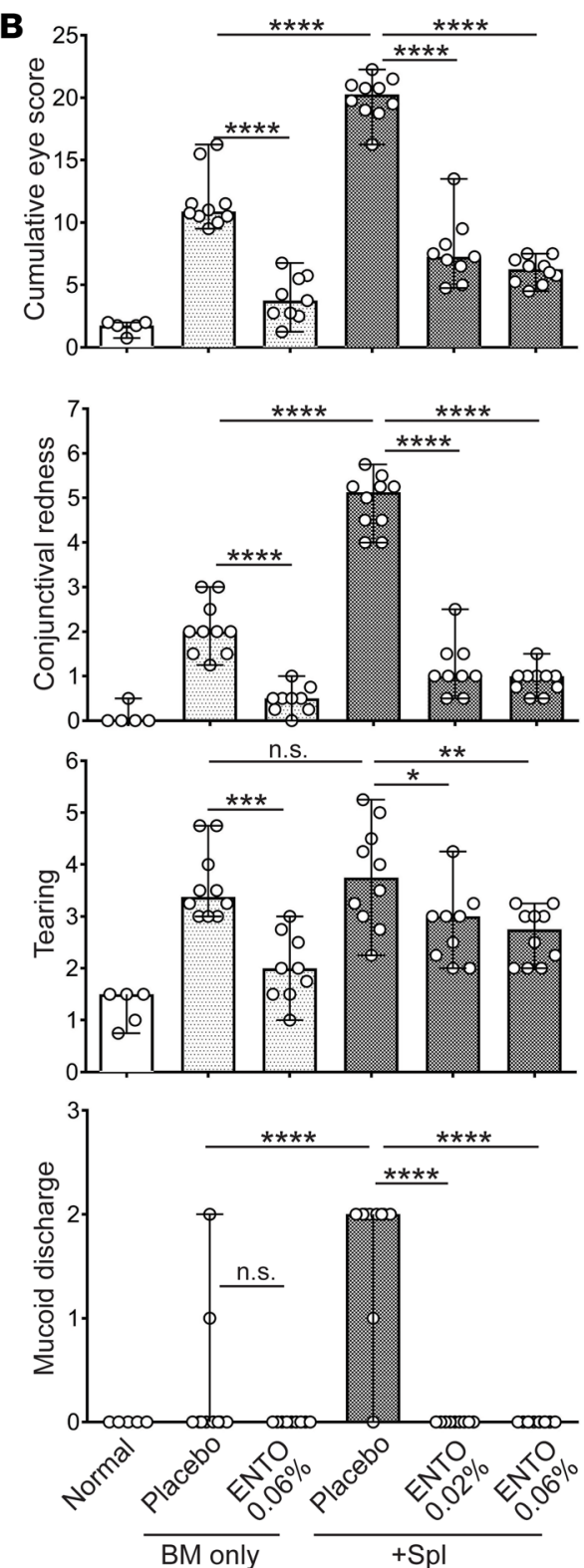

Figure 2. ENTO improves clinical eye scores in +Spl mice. (A) Representative images of the eye regions of mice from each HCT and ENTO/ placebo treatment group on day +33 . (B and $\mathbf{C}) 4$ weeks following transplant, all mice in each HCT group ( $n=9-10 /$ group) were evaluated for various clinical manifestations of eye pathology and scored in a masked fashion by an expert investigator specializing in models of eye disease in the mouse. Age-matched normal BALB/c mice ( $n$ $=5$ ) were included as reference healthy controls. (C) For chemosis, eyelid edema, conjunctival redness, and tearing, graded scoring by biomicroscopy for each eye ranged from 0 (not detectable) to 3.0 (most severe) as follows: 0 , none; $0.25-1.0$, mild; 1.25-2.0, moderate; and 2.25-3.0, severe. Blepharitis and mucoid discharge were scored as either being absent (scored as 0 ) or present (scored as 1). Each symbol in a particular graph represents the sum clinical scores of the right plus left eye of a single mouse. (B) Cumulative eye scores represent the sum of the following individual scores: chemosis, conjunctival redness, eyelid edema, and tearing. Each symbol represents an individual mouse. Bars indicate the median \pm range. Statistical analysis was performed by 1-way ANOVA with Tukey's multiple comparisons test (GraphPad Prism). ${ }^{*} P<0.05$; ${ }^{*} P<0.01$; ${ }^{* *} P<0.001$; ${ }^{* * *} P<0.0001$.

as described previously $(42,43)$. Our expert eye investigator was "masked" to all treatment groups. Normal, age-matched female BALB/c mice not receiving HCT were also included in the masked analysis as healthy controls. Cumulative eye scores (as defined in the legend for Figure 2B) were dramatically greater for placebo-treated GVHD mice relative to either ENTO-treated GVHD group $(P<0.0001$, Figure 2B). Placebo-treated GVHD mice had significantly higher pathologic eye scores for individual components of the eye score, including more severe chemosis, conjunctival redness, eyelid edema, tearing, blepharitis, and mucoid discharge, relative to either of the ENTO-treated GVHD groups $(P<0.0001$ for all comparisons, Figure $2 \mathrm{C})$. Tearing was also significantly greater in the $+\mathrm{Spl} /$ placebo group compared with either ENTO-treated GVHD group ( $P<0.05$ for $0.02 \%$ ENTO; $P<0.01$ for $0.06 \%$ ENTO, Figure $2 C$ ). In our transplant model, $\mathrm{BM}$-only control mice may receive a small number of residual $\mathrm{T}$ cells in the depleted $\mathrm{BM}$ product; this possibly accounts for placebo-treated BM-only control mice also having ocular manifestations compared with ENTO-treated BM-only control mice, as detectable by expert analysis under the microscope but not evident with the naked eye (Figure 2, B and C). Nevertheless, these findings were markedly less severe and some were absent in placebo-treated BM-only control mice compared with placebo-treated GVHD mice (Figure 2, B and C). Importantly, these masked clinical eye findings were consistent with those in a second, independent HCT experiment in which the donor T cell dose was reduced by one-fifth (a "four-fifths optimal" T cell dose, 
A $\underline{+S p l}$

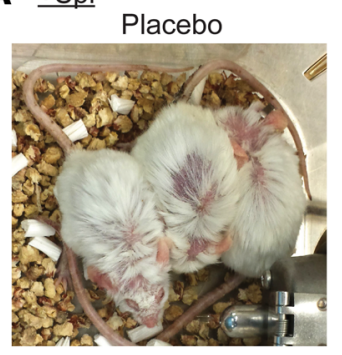

B

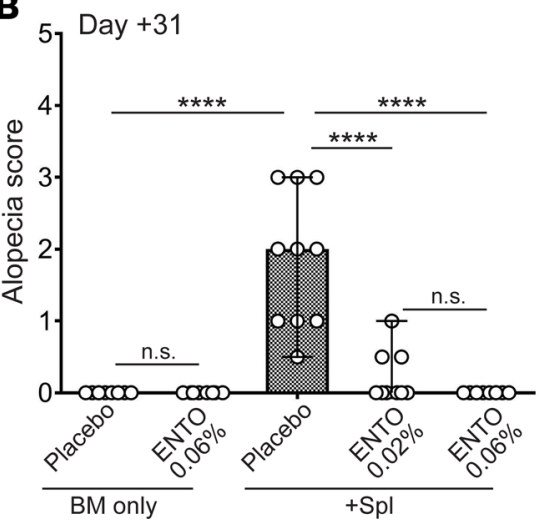

ENTO $0.02 \%$

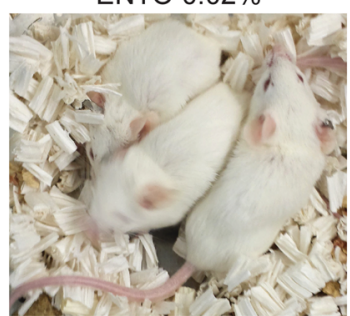

ENTO $0.06 \%$

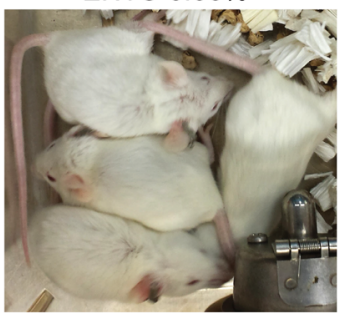

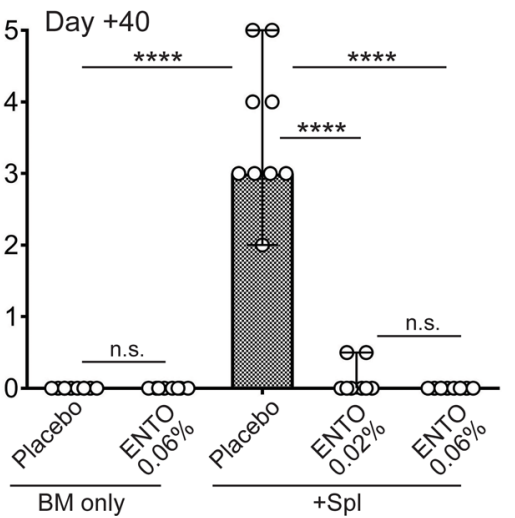

Figure 3. ENTO ameliorates alopecia in +Spl mice. (A) Images showing the coat status of representative mice from each $+5 p$ treatment group at day +40 . (B) All mice in each HCT group ( $n=9-10 /$ group) were evaluated in a masked fashion for severity of alopecia on day +31 and day +40 . Scores for individual mice were defined as follows: 0.5 , ruffling of fur; 1 , area of hair loss $<1 \mathrm{~cm}^{2} ; 2$, area of hair loss from $1-3 \mathrm{~cm}^{2} ; 3$, area of hair loss between $15 \%$ and $30 \%$ of body surface; 4 , area of hair loss between $30 \%$ and $50 \%$ of body surface; and 5 , area of hair loss $>50 \%$ of body surface. Each symbol represents an individual mouse. Bars indicate the median \pm range. Statistical analysis was performed by 1-way ANOVA with Tukey's multiple comparisons test (GraphPad Prism). ${ }^{* * *} P<0.0001$.

Supplemental Figure 2). As expected, combined clinical eye scores revealed an overall greater severity in HCT experiment 1 (optimal T cell dose, Figure 2) versus HCT experiment 2 (reduced T cell dose, Supplemental Figure 2). Nevertheless, ENTO treatment of GVHD mice significantly reduced eye score severity in both independent experiments.

Alopecia became readily apparent in most placebo-treated GVHD mice upon gross inspection by 1 month after HCT (Figure 3A). Mice were scored for alopecia severity by an investigator trained to assess GVHD skin findings who was masked to disease and treatment groups. The degree of alopecia was striking in placebo-treated GVHD mice compared with ENTO-treated GVHD mice at either dosage $(P<0.0001)$, with increasing severity from day +31 to day +40 (Figure 3B). By contrast, ENTO-treated GVHD mice remained generally free from alopecia development, except for mild alopecia observed in several mice in the ENTO-treated GVHD group receiving $0.02 \%$ drug (Figure 3B), suggesting an observable dose-dependent effect of this SYK inhibitor.

ENTO improved immune cell reconstitution in GVHD mice. Patients with superior HCT outcomes without GVHD generally experience robust immune cell recovery after HCT (23-26, 32, 44-47), which may be critical to the establishment of long-term immune tolerance $(23,25,26,32)$. To determine whether ENTO had an effect on immune cell reconstitution in our GVHD model, flow cytometry analysis of peripheral blood was performed to assess leukocyte frequency and the number in mice from all groups on day +28 (Figure 4A) and day +42 (Figure 4B) following HCT (16 days and 30 days after the initiation of ENTO, respectively). Remarkably, monocytes, B cells and T cells each recovered in significantly greater frequencies and numbers in ENTO-treated GVHD mice compared with placebo-treated GVHD mice at both time points. The frequencies and numbers of monocytes and $\mathrm{T}$ and B cells in GVHD mice was always statistically significant in the $0.06 \%$ ENTO group when compared with placebo-treated GVHD mice. Cell numbers either reached significance or trended higher at the $0.02 \%$ ENTO dose. MHC haplotype analysis by flow cytometry revealed that blood lymphocytes in all 
A Day +28: Monocytes
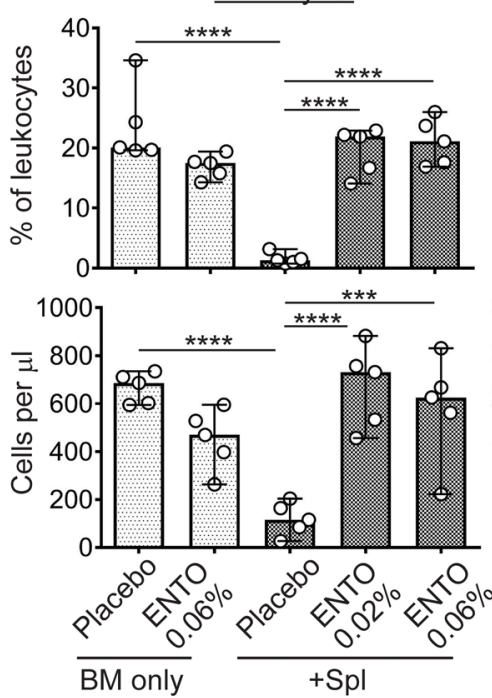

B Day +42 :
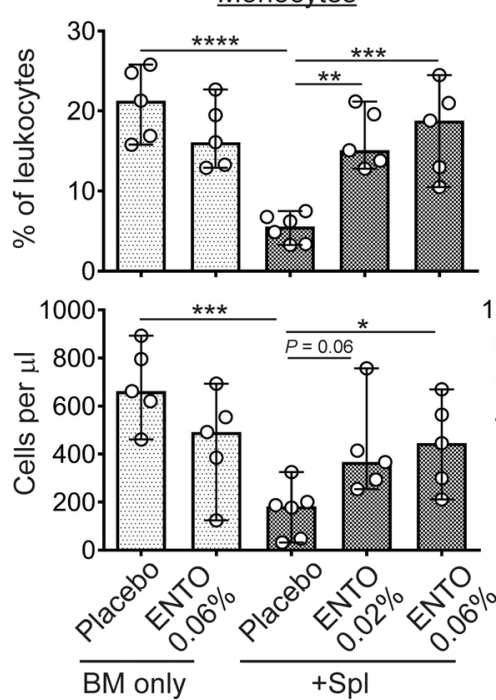
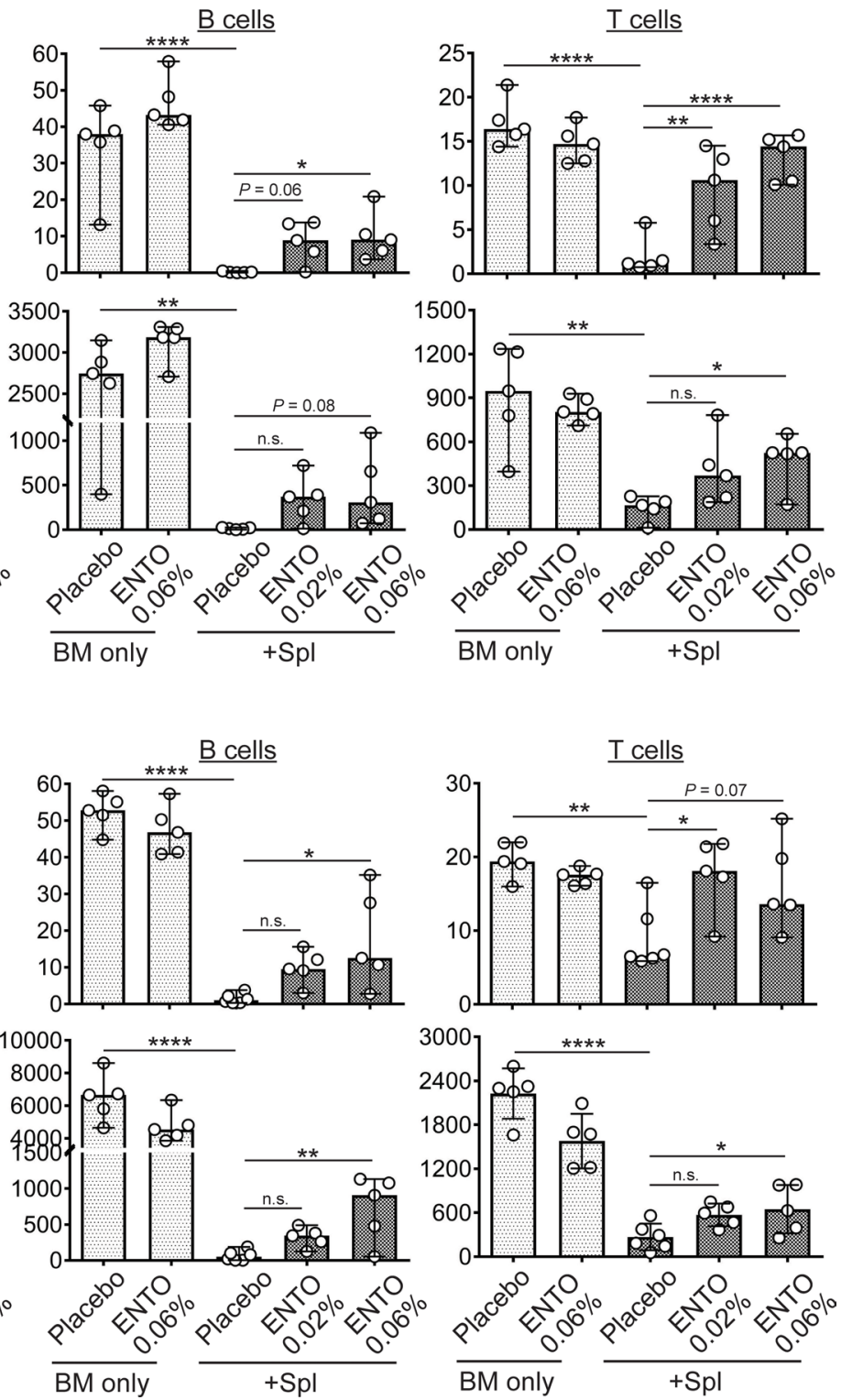

Figure 4. ENTO improves immune cell reconstitution in $+\mathbf{S p l}$ mice. Blood was obtained from representative mice in each HCT group on day +28 ( $n=5$, A) and day +42 ( $n=5-6, B)$, and flow cytometry analysis performed to evaluate monocyte, B cell, and T cell frequencies and numbers. Each symbol represents an individual mouse. Bars indicate the median \pm range. Statistical analysis was performed by 1-way ANOVA with Tukey's multiple comparisons test (GraphPad Prism). ${ }^{*} P<0.05 ;{ }^{*} P<0.01 ;{ }^{* *} P<0.001 ;{ }^{* * *} P<0.0001$. All mice in each HCT group $(n=9-10 /$ group) are represented either on day +28 or on day +42 .

3 GVHD groups were fully donor-derived (C57BL/6, $\mathrm{H} 2-\mathrm{Kb}^{+}$; Supplemental Figure 3). In the BM-only groups, B cells were also completely donor derived. Typical of mouse allo-HCT, some recipient $\mathrm{T}$ cells (BALB/c, $\mathrm{H} 2-\mathrm{Kd}^{+}$) remained in the BM-only groups, although the majority were also donor derived (Supplemental Figure 3). Thus, ENTO treatment was associated with increased blood immune cell recovery in GVHD mice.

ENTO promoted peripheral immune homeostasis by significantly decreasing pathological $B$ and $T$ cells and increasing Tregs. We previously demonstrated that pathogenic B cells are reliant on increased activation through SYK $(28,30)$, and others have shown that B cells drive pathogenic CD4+ T cells in mice with cGVHD manifestations (48). To address whether ENTO affected specific peripheral immune cell populations, we used flow cytometry to thoroughly phenotype the peripheral lymphocyte compartment in 5 GVHD group splenocyte samples taken at time of humane or study endpoint that could be compared with 5 control group samples. In humans and mice, antigen-activated B cells in the periphery can be identified by expression of the surface proteins CD27 and GL7, respectively $(24,26,32)$. Therefore, we used GL7 to identify activated 

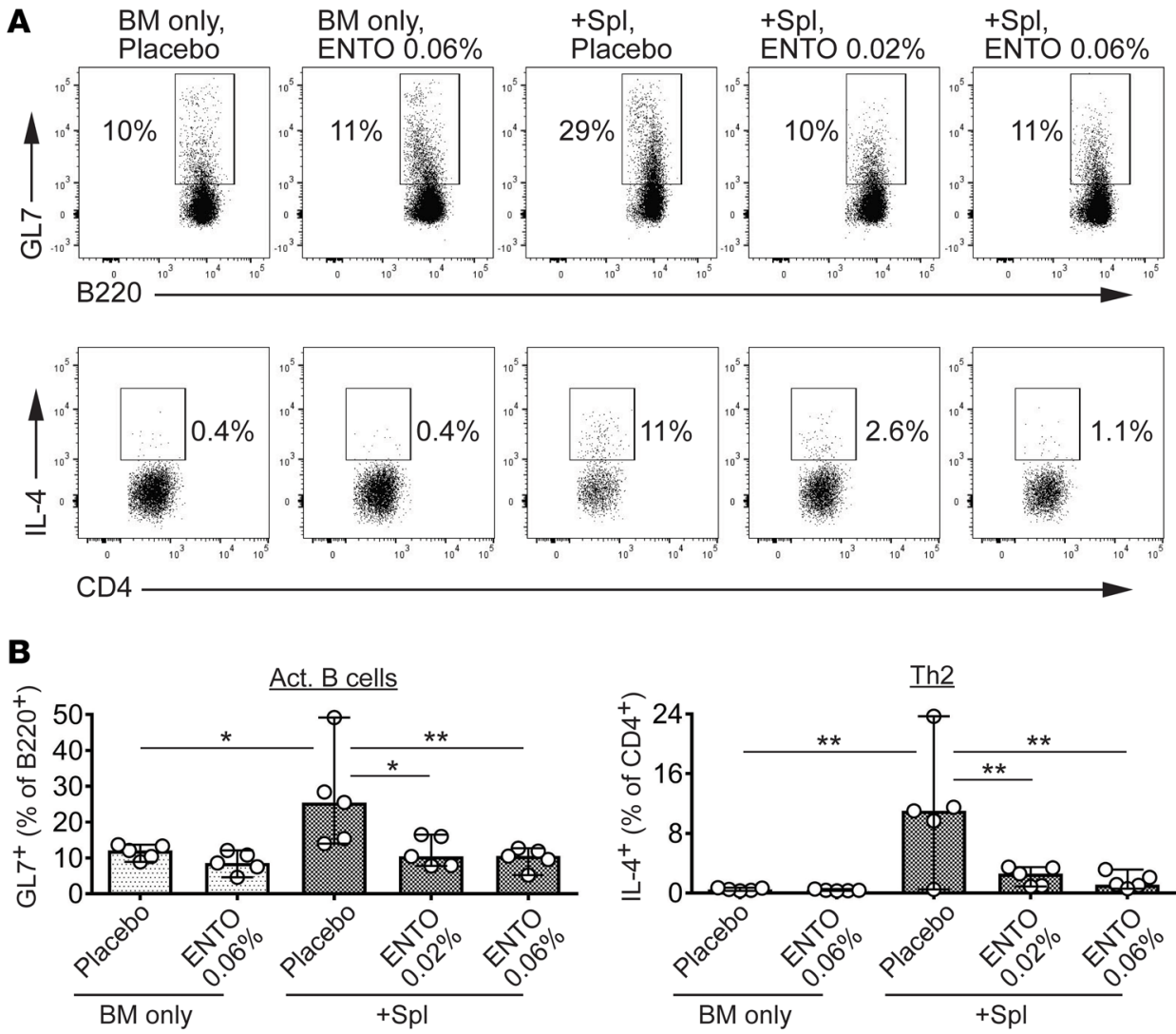

Figure 5. ENTO reduces activated B cells and Th2 cells in +Spl GVHD mice. (A) Representative flow cytometry plots showing gating strategies for GL7 expression by activated B cells and IL-4 expression by Th2 cells in splenocytes harvested from experimental mice and kept viably frozen until the time of analysis. To identify activated B cells, freshly thawed splenocytes were stained with B220 and GL7. To identify Th2 cells, splenocytes were cultured for 5 hours in medium in the presence of PMA and ionomycin and then surface stained for CD4, followed by intracellular staining for IL-4 (utilizing a Mouse BD Biosciences Th1/Th2/Th17 Phenotyping Kit). (B) Splenocytes from mice in all treatment groups ( $n=5$ each) analyzed for the subsets described in $\mathbf{A}$. Bars indicate the median \pm range. Statistical analysis was performed by 1-way ANOVA with Tukey's multiple comparisons test (GraphPad Prism). ${ }^{*} P<0.05 ;{ }^{* *} P<0.01$.

B cells in our GVHD model. We found that the activated GL7 ${ }^{+}$B cell subset was significantly increased in placebo-treated +Spl GVHD mice compared with placebo-treated control mice without GVHD (Figure 5), supporting previous studies showing increased activated B cells in the cGVHD condition in mice and humans $(5,19,20,23-26,28,31,32,49-52)$. Importantly, we observed a significant decrease in the proportion of GL7 $7^{+} \mathrm{B}$ cells in ENTO-treated GVHD mice compared with placebo-treated GVHD mice (Figure 5). As with GL7+ $B$ cells, the proportion of the IL-4-producing Th2 subset was also significantly increased in placebo-treated +Spl GVHD mice compared with placebo-treated BM-only control mice (Figure 5), and we also found a significant, concomitant decrease in Th2 cells in ENTO-treated +Spl GVHD mice (Figure 5). This is an interesting observation, since Th2 cells have a potential role in the propagation of both aGVHD and cGVHD (53-55). Thus, we found significant decreases in potentially pathologic $B$ and $T$ cells after ENTO treatment that associated with attenuation of GVHD.

We also examined additional important $\mathrm{T}$ cell subsets that have been associated with GVHD pathogenesis (56), including Tregs and Th1, Th17, and T follicular helper (Tfh) cells, in 5 GVHD group splenocyte samples taken at the time of humane or study endpoint that could be compared with 5 control group samples. These $\mathrm{T}$ cell subsets can be identified by the expression of well-defined transcription factors or cytokines (Figure 6A and Supplemental Figure 4). Tregs have previously been shown to attenuate disease in this mouse model (57). We observed a significant decrease in the frequency of Foxp $3^{+}$Tregs in placebo-treated +Spl GVHD mice compared with placebo-treated BM-only control mice (Figure 6), supporting the idea that a paucity of Tregs plays a role in cGVHD pathogenesis $(44,47,58-62)$. Remarkably, the frequency of Tregs was significantly increased or bordered significance in the ENTO-treated GVHD 

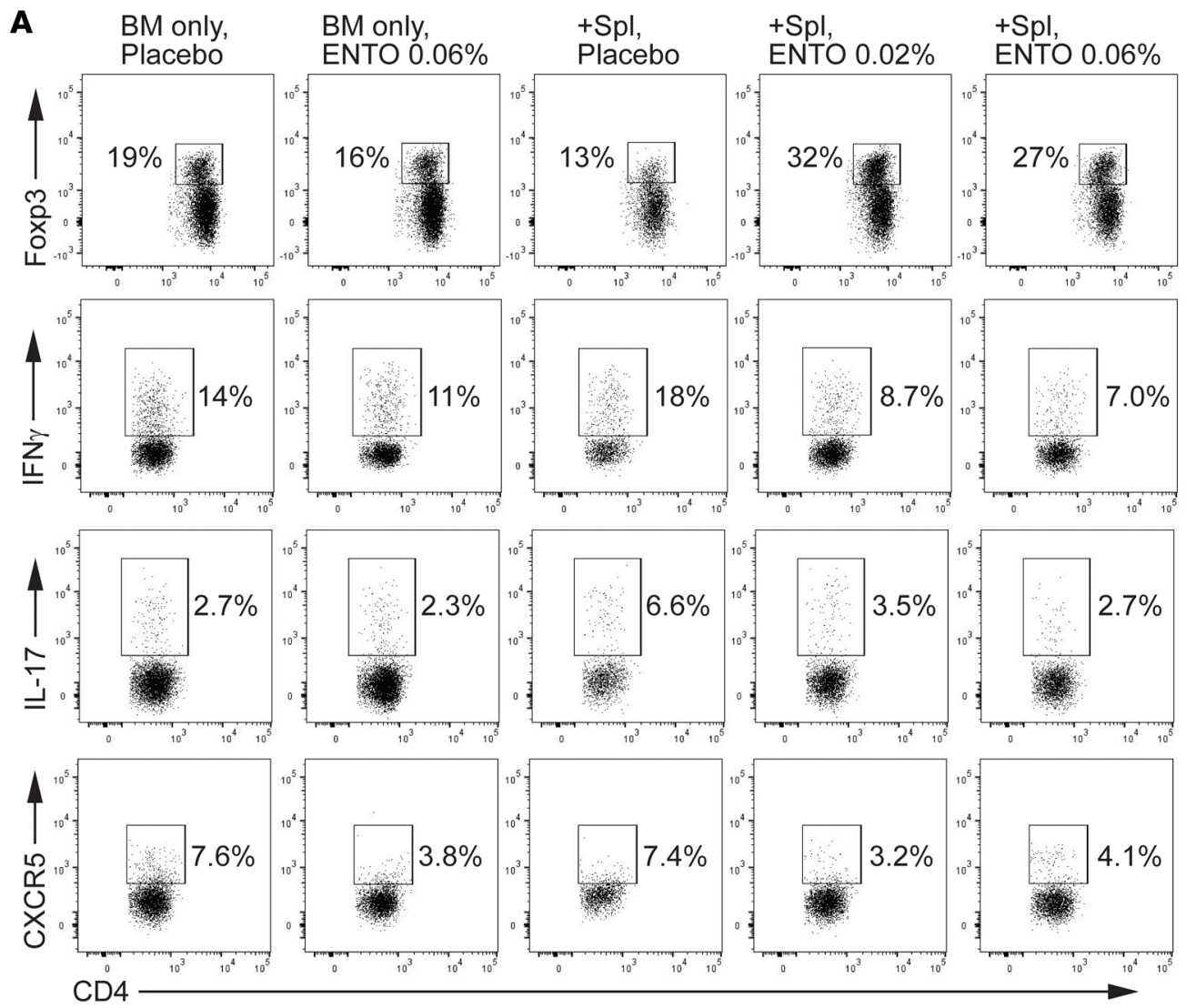

B
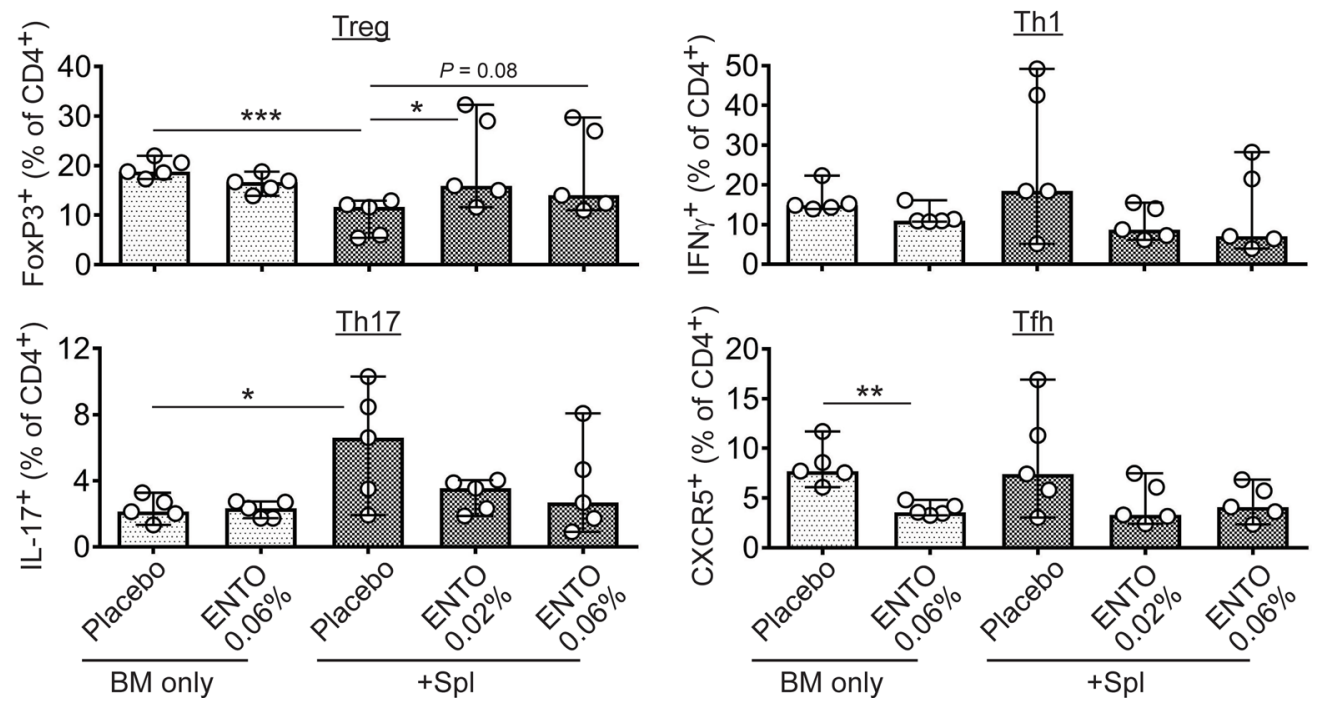

Figure 6. ENTO enhanced Tregs in +Spl GVHD mice. (A) Representative flow cytometry plots showing gating strategies for Foxp3 expression, cytokine production, and CXCR5 expression for the Treg, Th1, Th17, and Tfh subsets. To detect Tregs, splenocytes were surface stained for CD4, followed by intracellular staining for Foxp3. To detect Th1 and Th17 cells, splenocytes were cultured in the presence of PMA and ionomycin for 5 hours and then stained for surface CD4 and stained intracellularly for IFN- $\gamma$ or IL-17 (utilizing a Mouse BD Biosciences Th1/Th2/Th17 Phenotyping Kit). To detect Tfh cells, splenocytes were surface stained for CD4 and CXCR5. (B) Splenocytes from mice in all treatment groups ( $n=5$ each) analyzed for the subsets described in $\mathbf{A}$. Bars indicate the median \pm range. Statistical analysis was performed by 1-way ANOVA with Tukey's multiple comparisons test (GraphPad Prism). ${ }^{*} P<0.05 ;{ }^{*} P<0.01 ;{ }^{* *} P<0.001$.

groups compared with that in placebo-treated GVHD mice (Figure 6). We also found that the proportion of IL-17-producing Th17 cells was significantly increased in placebo-treated GVHD mice compared with that in placebo-treated control mice (Figure 6B), but there was no significant decrease in the proportion of Th17 cells with ENTO treatment. There was also no significant difference in IFN- $\gamma$-producing Th1 
cells among experimental groups (Figure 6B). Assessment of CXCR5 ${ }^{+}$Tfh cells revealed no significant difference among +Spl GVHD groups, although, notably, BM-only mice receiving ENTO treatment had a significant reduction in the frequency of Tfh cells compared with BM-only mice receiving placebo (Figure $6 \mathrm{~B})$. Together, these data show that the peripheral $\mathrm{T}$ cell compartment is influenced by ENTO. The mechanism underpinning these changes in T cells is likely indirect and requires further study.

Finally, because SYK is expressed in myeloid lineage cells and we found that monocytes recovered more readily in GVHD mice receiving ENTO (Figure 4), we examined myeloid subsets (Supplemental Figure 5). Monocytic myeloid-derived suppressor cells (M-MDSCs) are a unique myeloid subset with regulatory properties, identified phenotypically in mice as $C D 11 b^{+} L y 6-C^{\text {hi }} L y 6-G^{-}(63)$. Interestingly, the frequency of M-MDSCs was significantly higher in placebo-treated +Spl GVHD mice compared with that in placebo-treated BM-only control mice (Supplemental Figure 5), suggesting that M-MDSCs may be generally increased within the myeloid lineage in response to the cGVHD condition. We found that the frequency of M-MDSCs was similar between all 3 +Spl GVHD treatment groups (Supplemental Figure 5), suggesting this cell population was not affected by ENTO.

ENTO prolonged the survival of GVHD mice. Mice were closely monitored from the time of HCT for a total of 120 days and sacrificed if predefined, Institutional Animal Care and Use Committee-approved humane endpoint criteria were reached. ENTO-treated GVHD mice had a significant increase in life span compared with placebo-treated GVHD mice, with 60\% (6 of 10) surviving in each ENTO group versus $10 \%$ (1 of 10) surviving in the placebo group (Figure 7A, top). Survival curves were similar for ENTO-treated GVHD groups at either drug dose (Figure 7A, bottom left), demonstrating a remarkable effect of ENTO on survival. Importantly, ENTO itself did not negatively effect survival, as life spans were similar for both BM-only groups (Figure 7A, bottom right).

A single surviving mouse in the placebo-treated GVHD group at day +120 did not reach humane endpoint criteria, enabling study endpoint assessment of abnormal tissues surrounding the ears and eyes (Figure 7B, top). Eyelid hyperplasia was apparent in this mouse compared with representative mice from each ENTO-treated GVHD group and both BM-only groups, as assessed by Masson's trichrome staining of eyelid sections after the mice were sacrificed on day +120 (Figure $7 \mathrm{~B}$, bottom).

The reduced survival rate of placebo-treated GVHD mice was likely due to the cGVHD condition rather than a higher rate of infections, since no mice manifested gross or histopathological evidence of infection and mice in all groups maintained natural IgM antibodies in their plasma reactive against vaccinia virus (VV; Supplemental Figure 6). We utilized VV as our model pathogen since it was shown previously by Zinkernagel's group that natural IgM antibodies in mice are required to prevent the spread of VV and other pathogens to vital organs (64). Notably, with the exception of one outlier, natural IgM levels trended higher in ENTO-treated GVHD mice compared with those in placebo-treated GVHD mice (Supplemental Figure 6), likely reflecting the significantly increased B cell frequencies and numbers observed in ENTO-treated GVHD mice (Figure 4).

ENTO improved pathological skin scores and reduced the presence of dermal SYK cells in GVHD mice. To determine whether skin GVHD was attenuated by ENTO, histopathological analysis was performed in a masked fashion by an expert from the Duke Department of Pathology who specializes in transplant-related diseases, including GVHD. Prominent pathological skin findings occurring in placebo-treated GVHD mice were less evident in ENTO-treated GVHD mice. Specifically, dermal thickening caused by increased collagen abundance along with a paucity of hair follicles, each occurring in placebo-treated GVHD mice, were normalized in ENTO-treated GVHD mice (Figure 8A, stars indicate areas of dense collagen; arrows indicate normal hair follicle structures). Clinical dermis scores, defined as GVHD-associated increases in collagen density on a relative scale (detailed in the legend for Figure 8), were significantly less severe in the ENTO $0.02 \%$ GVHD group, and scores trended toward lesser severity in the ENTO $0.06 \%$ GVHD group compared with placebo-treated GVHD mice (Figure 8B). Likewise, follicle scores, defined as a paucity in the number of hair follicles per defined area (detailed in the legend for Figure 8), were significantly improved in both groups of ENTO-treated GVHD mice compared with placebo-treated GVHD mice (Figure 8B). Epidermis and inflammation skin scores were also either significantly improved or trended toward normalcy in ENTO-treated GVHD mice (Figure $8 \mathrm{~B})$. Total skin scores (the 4 criteria described above combined), which highlight individual mice with multiple pathologic skin symptoms, were also significantly reduced in both groups of ENTO-treated GVHD mice compared with placebo-treated GVHD mice (Figure 8B). 
A
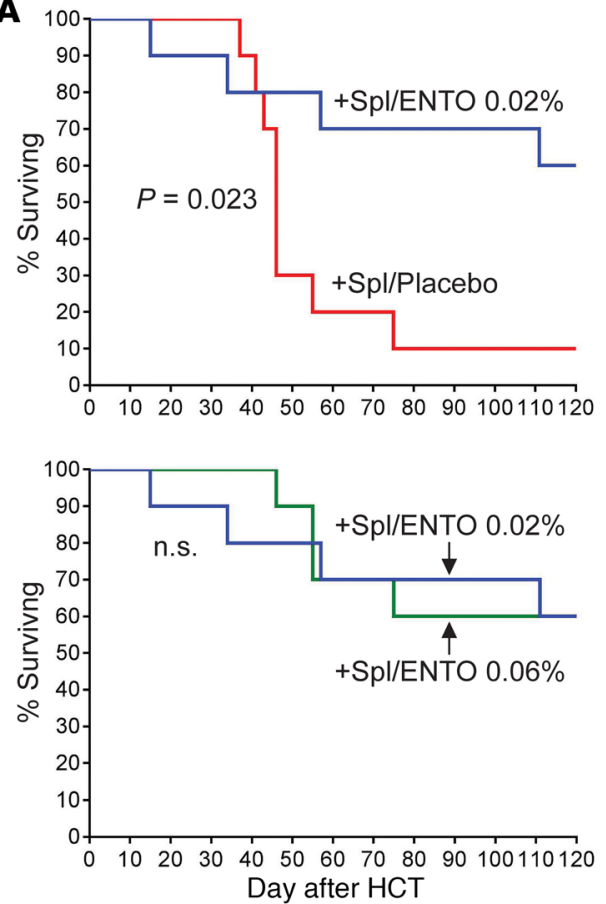

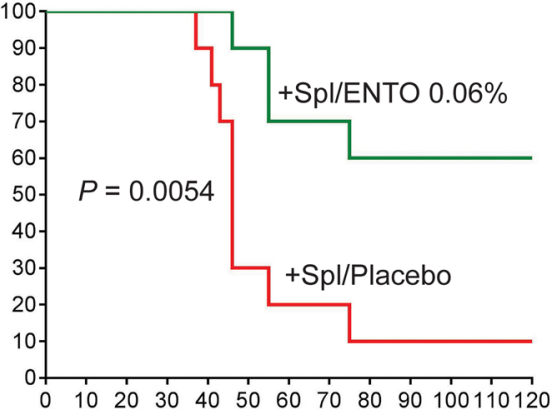

BM only/Placebo

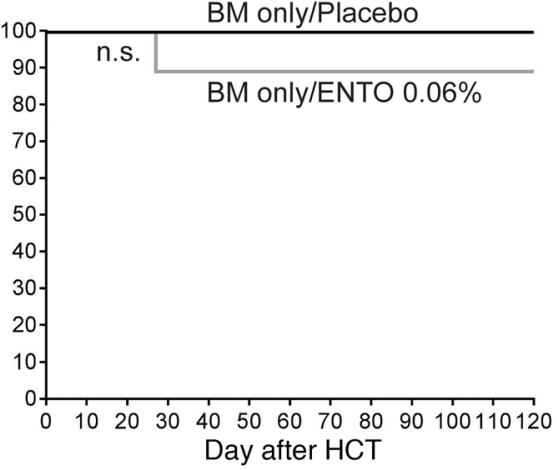

Figure 7. ENTO prolongs the survival of $+5 p$ mice. (A) Data represent Kaplan-Meier plots comparing the survival of mice in all HCT groups ( $n=9-10 /$ group) through day +120 . Statistical analysis was performed between the groups shown using the log-rank test (GraphPad Prism). $P$ values reaching statistical significance are as indicated. (B) Top: Day +120 images of the single surviving $+\mathrm{Spl} /$ placebo group mouse, along with images of representative mice from all other groups. Bottom: Masson's trichrome staining of eyelid tissue, showing marked hyperplasia in the surviving + Spl/placebo mouse compared with representative mice from all other groups. Scale bar: $115 \mu \mathrm{m}$

\section{B}

B Day +120 BM only
\[ \begin{array}{cc}\text { Placebo } & \text { ENTO } 0.06 \% \\ \text { (representative) } & \text { (representative) }\end{array} \]

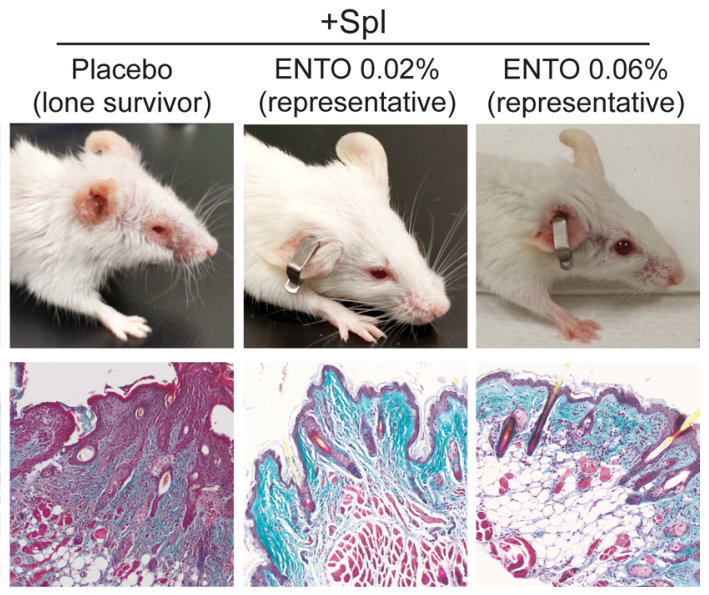

Immunohistochemical analysis of SYK expression in the skin revealed an abundance of SYK ${ }^{+}$cells that localized to the dermal and subdermal regions of placebo-treated GVHD mice (Figure 9A, brown cells). By contrast, SYK ${ }^{+}$cells were generally absent in ENTO-treated GVHD mice at either dosage (Figure 9A). BM-only group mice (both placebo and ENTO treated) also lacked these $\mathrm{SYK}^{+}$cells in the skin (data not shown). Interestingly, these cells closely resembled $\mathrm{SYK}^{+}$cells localizing to lichenoid skin lesions of cGVHD patients, where B cells can also be found in these relatively nucleated cell rich lesions (Supplemental Figure 7). Immunohistochemical analysis of serial skin sections from placebo-treated GVHD mice revealed that $\mathrm{SYK}^{+}$cells were either B220+ or F4/80+ (Figure 9B, top, and data not shown), indicating the capacity of both $\mathrm{B}$ cells and macrophages, respectively, to reside at these inflammatory sites in the skin. Some B220 $\mathrm{B}$ cells in the skin had the morphology of plasmablasts/plasma cells with eccentric nuclei (Figure 9B, bottom left), and $\mathrm{F} 4 / 80^{+}$macrophages had a typical, relatively large morphologic appearance (Figure 9B, bottom right).

ENTO induces a greater rate of apoptosis in B cells from HCT patients with active $G$ GHD compared with patients without $c G V H D$. It was previously shown that the exposure of B cells isolated from HCT patients with active cGVHD to fostamatinib for 48 hours induced significantly greater apoptosis compared with fostamatinib exposure of B cells isolated from patients with no cGVHD (28). To test whether ENTO preferentially affected B cells from patients with active cGVHD, purified B cells were treated ex vivo with ENTO for 48 hours. 
A

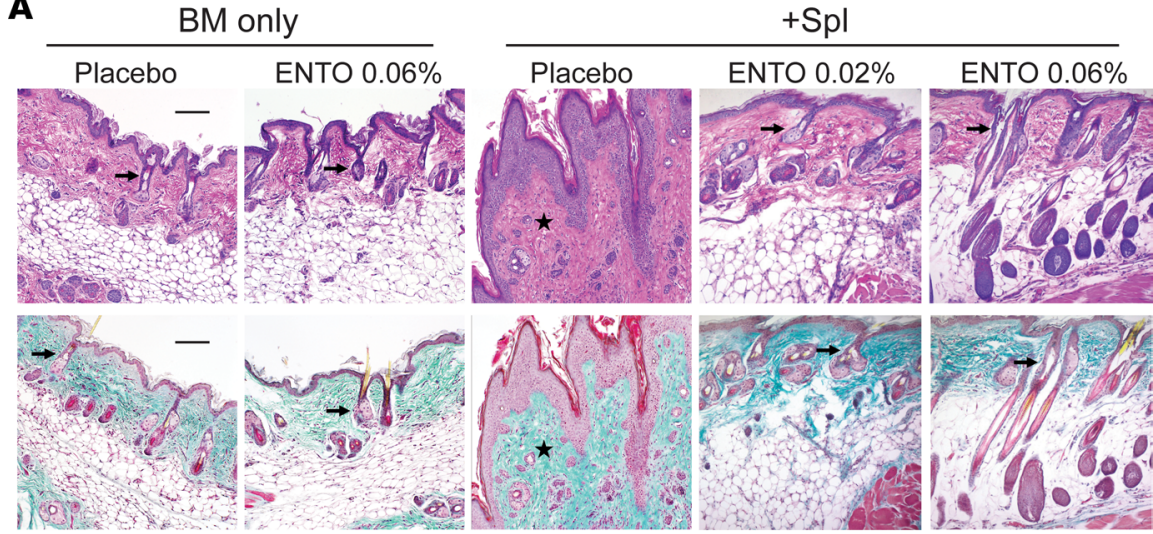

B
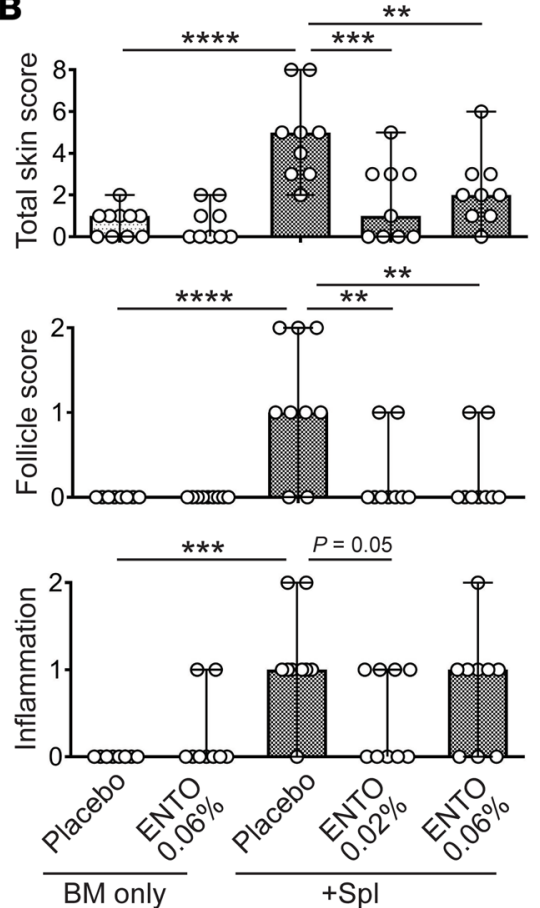
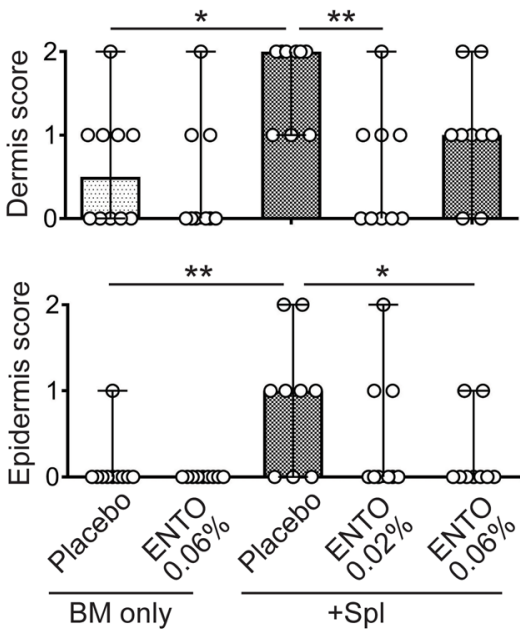

Figure 8. ENTO reduces skin GVHD in +Spl mice.

(A) Representative images of skin sections obtained from the upper backs of mice from the indicated groups stained with $\mathrm{H} \& \mathrm{E}$ (top) and Masson's Trichrome (bottom). Stars indicate regions of increased collagen density, indicative of GVHD-mediated fibrosis, in +Spl/placebo mice. Arrows indicate hair follicles, which were largely absent in + Spl/placebo mice. Scale bar: $115 \mu \mathrm{m}$. (B) Various skin disease criteria from all mice in each HCT group ( $n=9-10 /$ group), scored in a masked fashion by an expert pathologist specializing in GVHD-mediated skin disease. Scoring criteria were as follows: dermis ( 0 , normal; 1 , mild increased collagen density; 2 , marked increased collagen density); hair follicles ( 0 , normal number of approximately 5 per linear millimeter; 1 , between 1 and 5 follicles per linear millimeter; $2,<1$ follicle per linear millimeter); epidermis ( 0 , normal; 1 , interface damage in $<20 \%$ of section with occasional necrotic keratinocytes; 2 , widespread interface damage ( $>20 \%$ of section); inflammation ( 0 , none; 1 , focal infiltrates; 2 , widespread infiltrates). Each symbol represents an individual mouse. Bars indicate the median \pm range. Total skin score represents the sum of all above criteria for each mouse. Statistical analysis was performed by 1-way ANOVA with Tukey's multiple comparisons test (GraphPad Prism). ${ }^{*} P$ $<0.05 ;{ }^{* *} P<0.01 ;{ }^{* * *} P<0.001 ;{ }^{* * *} P<0.0001$.

Significantly higher levels of apoptosis were found in ENTO-treated active cGVHD B cells compared with ENTO-treated B cells from HCT patients without cGVHD (Figure 10A). This effect was prominent at concentrations within the range of the ENTO $\mathrm{IC}_{50}$ value (Figure 10B). Interestingly, at this concentration range, increased apoptosis by ENTO in the active cGVHD setting was selective to B cells, as the effect was not observed in either T cells or monocytes (Figure 10C). These data suggest that SYK inhibition by ENTO may favor the elimination of hyperactivated, potentially pathogenic B cells through apoptosis.

\section{Discussion}

In this study, oral administration of the SYK inhibitor ENTO to mice early after HCT blocked development of clinical manifestations of eye and skin GVHD and enhanced survival. ENTO binds within the kinase domain of SYK, occupying the ATP binding pocket and inhibiting the capacity of SYK to phosphorylate downstream substrates on regulatory tyrosines (41). We found that proportions of peripheral GL $7^{+} \mathrm{B}$ cells and Th2 cells increased in GVHD and these were significantly decreased after early treatment with ENTO. The clinical improvement in mice was also associated with decreases in $\mathrm{SYK}^{+} \mathrm{B}$ cells and macrophages in lesional tissues. Remarkably, these significant decreases in the proportions of cells known to be involved in GVHD pathogenesis was accompanied by an associated improvement in immune cell recovery and homeostasis after ENTO treatment in GVHD mice. 
A Stain: SYK
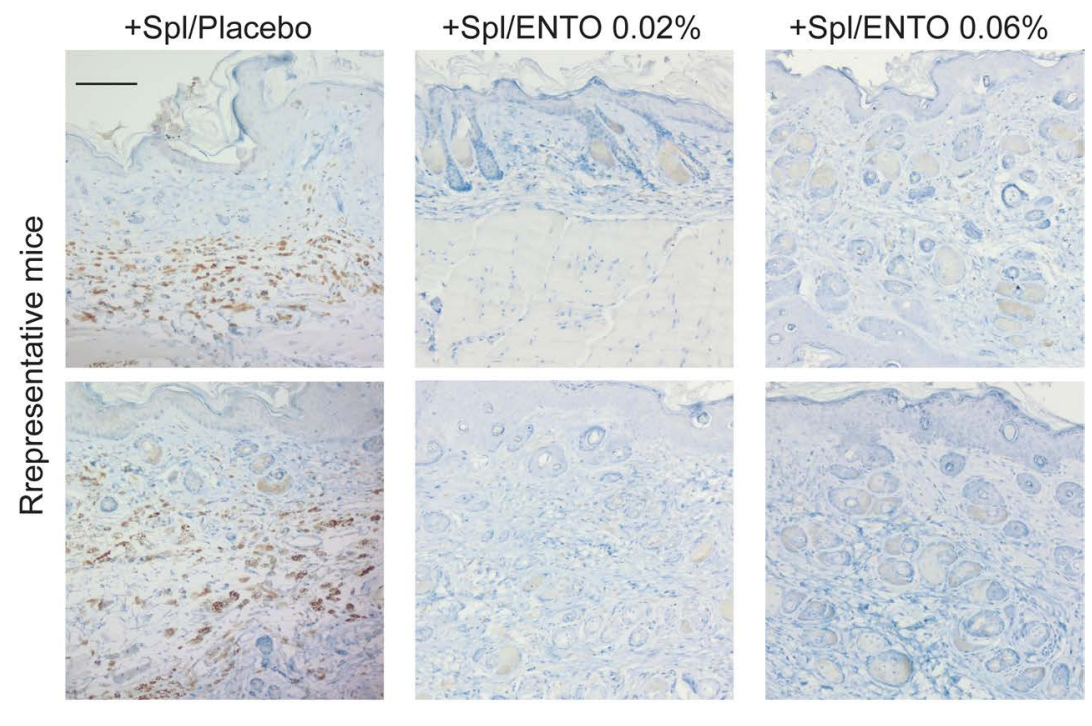

B

+ Spl/Placebo (serial sections)
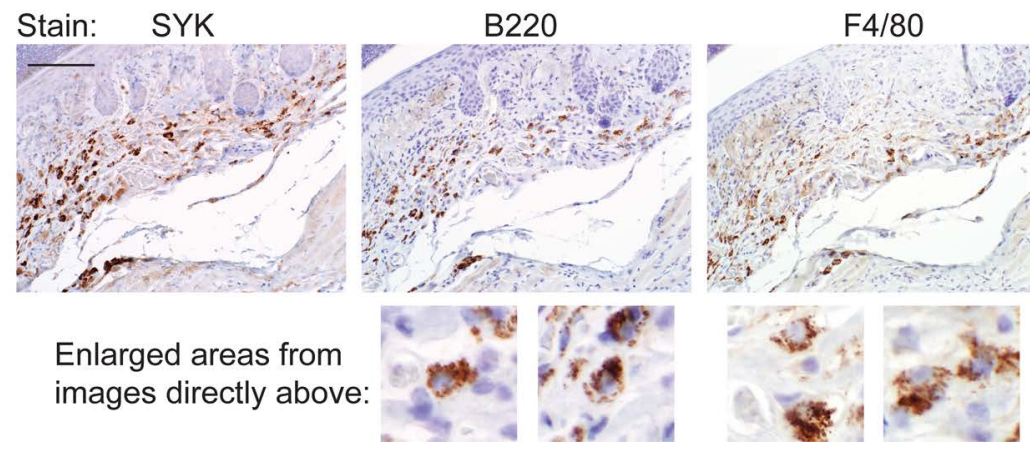

Figure 9. Dermal SYK' cells are prevalent in +Spl/placebo mice and absent in +Spl/ENTO mice. Immunohistochemistry was performed on FFPE skin sections obtained from the upper backs of mice from the indicated HCT treatment groups. (A) Skin sections from 2 representative mice from each treatment group stained with anti-SYK Ab (brown) and counterstained with hematoxylin (blue), as described in the Methods section. Scale bar: $115 \mu \mathrm{m}$. (B) Top: Serial skin sections from a representative + Spl/placebo mouse were stained with anti-SYK Ab, anti-B220 Ab, or anti-F4/80 Ab. Scale bar: $115 \mu \mathrm{m}$. Images in the bottom row are shown to highlight the morphology of representative cells staining positive for B220 (left 2 images) or F4/80 (2 right images), enlarged from images in the top row. Each panel on the bottom row represents a width of $42 \mu \mathrm{m}$.

ENTO has also demonstrated efficacy and an acceptable safety profile in a phase II clinical trial for the treatment of CLL and other non-Hodgkin lymphoma patients, without adverse effects on immune suppression in patients (65), but how it affects immune recovery after HCT was unknown prior to our studies described herein. SYK is expressed in multiple immune cell types that may contribute to cGVHD pathogenesis after HCT, including monocytes/macrophages, B cells, neutrophils, basophils, and mast cells (41). Thus, multiple cell types expressing SYK may be important for ENTO efficacy in mitigating cGVHD. The mechanism(s) by which specific SYK inhibition may be efficacious in treating cGVHD, while not inducing global immune suppression in any particular cell lineage expressing SYK, is important and intriguing. Maintenance of immune homeostasis in the presence of ENTO treatment does in fact appear to be the case, as no adverse infectious events or lymphopenia were observed in healthy volunteers in a multiple ascending-dose study of ENTO to evaluate safety, pharmacokinetics, and pharmacodynamics (66). Despite the fact that monocytes, B cells, and T cells each have clear roles in CGVHD pathogenesis, each of these subsets occurred in significantly greater frequencies and numbers in ENTO-treated GVHD mice compared with placebo-treated GVHD mice (Figure 4). This improved immune cell reconstitution with ENTO compared with other agents may relate in part to its known target selectivity profile. Bioassays assessing the activity of ENTO in side-by-side comparisons with another SYK inhibitor, fostamatinib (R406), showed 
A

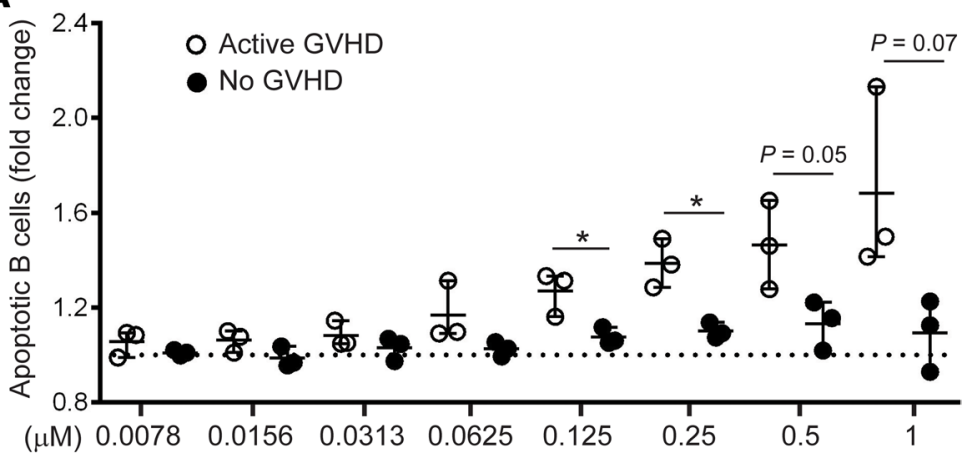

B
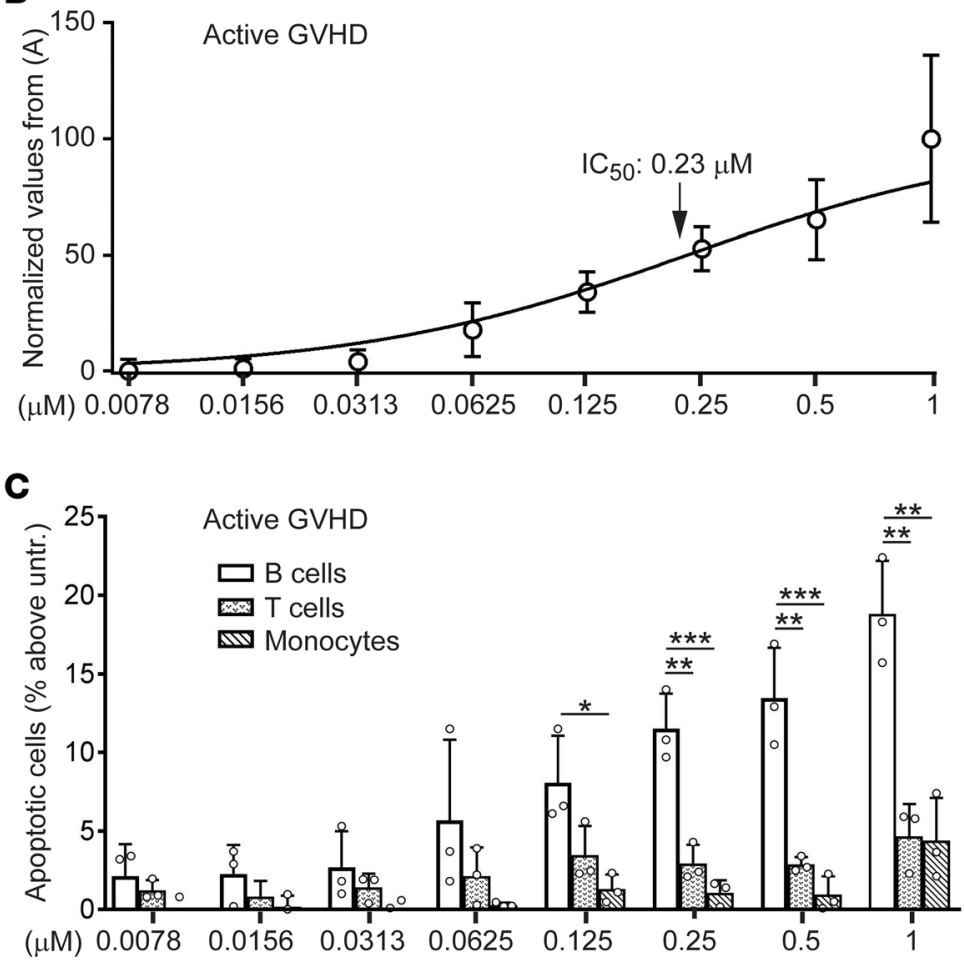

Figure 10. Syk inhibition by ENTO selectively augments the apoptosis of B cells from patients with active cGVHD. (A) PBMCs from patients with active CGVHD ( $n=3$, white circles) or no cGVHD ( $n=3$, black circles) were cultured for 48 hours in the presence of 2 -fold increasing concentrations of ENTO ranging from 0.0078 to $1 \mu \mathrm{M}$. The cells were then harvested and analyzed by flow cytometry for the frequency of apoptotic B cells (CD19+Annexin $V^{+}$7-AAD-). B cell apoptosis at each concentration is represented as the following ratio: \%Annexin $\mathrm{V}^{+} / 7-\mathrm{AAD}^{-} \mathrm{B}$ cells (ENTO treated)/\%Annexin $\mathrm{V}^{+} / 7-\mathrm{AAD}^{-} \mathrm{B}$ cells (untreated). Values are shown as mean with range. Statistical analysis comparing the ratios of apoptotic B cells between the active and inactive CGVHD groups was performed using a 2-tailed, unpaired Student's $t$ test (GraphPad Prism). (B) Graphic display and curve fit analysis with determination of the $\mathrm{IC}_{50}$ for ENTO apoptosis-inducing activity in active cGVHD B cells was performed using GraphPad Prism software on the active CGVHD B cell data in $\mathbf{A}$. Values are shown as mean \pm SEM. (C) The frequency of B cells, T cells, and monocytes from active cGVHD patients ( $n$ = 3) undergoing apoptosis in the presence of ENTO, across the concentration range described in $\mathbf{A}$, that was above the frequency of apoptotic cells for each population treated with vehicle alone. Values are shown as mean \pm SD. Statistical analysis was performed by 1-way ANOVA with Tukey's multiple comparisons test (GraphPad Prism). ${ }^{*} P<0.05 ;{ }^{* *} P<$ $0.01 ;{ }^{* *} P<0.001$

some striking differences between these agents. In TF-1 cells stimulated with EPO, for example, where JAK2-mediated phosphorylation of STAT5 is the readout, the $\mathrm{EC}_{50}$ for R406 inhibitory activity toward JAK2 was 35-fold less than that of ENTO (39). Likewise, in MV4-11 cells, which undergo FLT3-dependent proliferation, the $\mathrm{EC}_{50}$ for R406 inhibitory activity toward FLT3 was 33-fold less than that of ENTO (39). Similar results were observed for other kinases, including c-Kit, RET, and KDR. Furthermore, in other 
bioassays using BCR-stimulated primary B cells and B cell lines, where the readouts included SYK-dependent BLNK phosphorylation, proliferation, and CD86 expression, the $\mathrm{EC}_{50}$ for ENTO inhibitory activity toward SYK was always lower than that of R406 by 2- to 4-fold (39). Collectively, our data and the known superior selectivity and concentration profile of ENTO for SYK provide insight into various clinical aspects of CGVHD that are dependent on SYK activity and that may be safely averted in patients if treated early after transplant with ENTO.

Recent studies have defined a role for B cells in the pathogenesis of cGVHD, both in humans and in mouse models of the disease $(17,19-21,24,32)$. Paradoxically, patients who do not go on to develop cGVHD recover B cell numbers faster within the first year following HCT compared with patients that develop cGVHD, who have persistently low B cell numbers $(25,26,32)$. Likewise, patients who recover B cells in greater numbers after completing regimens of B cell depletion therapy with rituximab have a significantly reduced incidence of cGVHD recurrence or severity of disease (23). This apparent paradox between greater B cell numbers and diminished cGVHD appears to be explained by the fact that, in an allogeneic environment where donor B cell numbers remain low, high levels of the B cell survival factor BAFF cause alloantigen-activated (alloAg-activated) $\mathrm{CD} 27^{+} \mathrm{B}$ cells to survive and, as a consequence, produce pathogenic alloAbs, act as alloAg-presenting cells, or both $(24-26,32)$. In addition, IL-10-producing regulatory B cell numbers are significantly reduced in patients with active cGVHD (67), suggesting that a certain level of regulatory B cells may be optimal to suppress potentially pathogenic $\mathrm{T}$ cells and to maintain immune tolerance. Therefore, therapeutics that suppress or eliminate alloAg-activated B cells but that do not eliminate normal B cells may be highly efficacious in cGVHD treatment. The possibility that ENTO may selectively target pathogenic B cells in patients with cGVHD remains intriguing, since this would potentially eliminate destructive B cells in favor of B cells that would function in normal humoral immune responses against bacteria, fungi, and viruses that commonly cause morbidity and mortality in cGVHD. In support of this, ENTO reduced the frequency of activated GL7 $7^{+}$B cells in the spleens of GVHD mice when compared with GVHD mice receiving placebo (Figure 5). Also supporting this concept, ENTO induced a greater level of apoptosis of B cells from HCT patients with active cGVHD compared with HCT patients with no cGVHD (Figure 10). Furthermore, ENTO-treated mice harbored natural IgM antibodies reactive with VV as model pathogen (Supplemental Figure 6). These antibodies are critical in providing natural protective immunity to common pathogens (64), and no apparent infections occurred in any ENTO-treated mice in our studies. This further suggests that ENTO may target pathogenic, hyperactivated B cells for apoptosis, while sparing more quiescent B cells that can participate in normal immune responses.

In addition to attenuation of an activated $\mathrm{B}$ cell subset, we also found that peripheral $\mathrm{T}$ cell homeostasis improved after ENTO. The frequency of Tregs was enhanced in GVHD mice receiving ENTO (Figure 6), while the frequency of Th2 cells was decreased (Figure 5) compared with GVHD mice receiving placebo. We believe this was an indirect effect, since we know that ENTO has a 30-fold greater selectivity for SYK than for its closest tyrosine kinase ortholog that is primarily restricted to T cells, ZAP-70 (41). In fact, in DiscoverX KINOMEscan assays designed to evaluate the specificity of small-molecule inhibitors under development toward $>350$ kinases, only 1 kinase fell within 10-fold selectivity relative to SYK selectivity by ENTO (TNK1, 8-fold less selectivity, refs. 39, 41). By contrast, in these same assays comparing ENTO and R406 (fostamatinib) side by side, 36 kinases fell within 10-fold selectivity by R406 when compared with SYK, and, remarkably, one-third of these kinases exhibited even greater selectivity by R406 than for SYK itself (39). Thus, the high selectivity and affinity of ENTO for SYK suggests that the observed effects in our mouse model are due to SYK blockade and not significant off-target effects toward other kinases. The mechanisms by which ENTO likely indirectly affects the peripheral T cell compartment represent an interesting avenue for exploration in future studies.

Collectively, our observations further support existing evidence that immune homeostasis is beneficial not only in maintaining GVL responses and preventing initial disease relapse, but also in improving the likelihood that cGVHD will not occur $(23,25,26,32,46$, 47). By contrast, in HCT patients with a lymphopenic environment, pathogenic immune cells of various lineages may emerge as a consequence of constant exposure to alloAg and cytokines such as BAFF that are more abundant on a per cell basis, resulting in cGVHD (25, $26,32,46)$. Furthermore, it is likely that these pathogenic immune cells emerge more rapidly in some HCT patients due to intrinsic signaling defects that are exacerbated due to continual exposure of developing cells to AlloAg and cytokines. Examples of this scenario in cGVHD include a paucity in Tregs and regulatory B cells $(46,60,62,67,68)$, an increase in M2-like macrophages that express CSF-1R and invade the skin causing fibrosis (38), and B cells that have dysregulated expression of the key transcription factors IRF4 and IRF8, 
causing heightened signaling through $\mathrm{NOTCH} 2$, which synergizes with the BCR to induce abnormally high proliferative responses to limiting amounts of antigen (52). Emerging evidence suggests that some of these intrinsic defects that drive immune cell pathogenicity can be corrected pharmacologically, steering developing immune cells down either regulatory pathways or more mature functional pathways $(3,9,45,47,52,69)$.

Agents that temper pathogenic immune cell responses by altering signaling pathways without broad killing and elimination of immune cells have the potential to prevent GVHD by establishing normal immune homeostasis, tolerance, and functionality. Our findings, along with the known safety profile of ENTO $(41,66)$, suggest we may now have another important tool for GVHD prophylaxis.

\section{Methods}

$H C T$. BALB/c recipient mice (The Jackson Laboratory) were lethally irradiated with 8.5 Gy using a Cesium 137 irradiator (Duke University). On the same day, irradiated mice were transplanted with $1 \times 10^{7} \mathrm{~T}$ cell-depleted C57BL/6 mouse (The Jackson Laboratory) BM cells alone (control HCT groups, i.e., BM only) or with $1 \times 10^{7} \mathrm{~T}$ cell-depleted C57BL/6 BM cells plus $1 \times 10^{6}$ C57BL/6 splenocytes (cGVHD groups, i.e., $+\mathrm{Spl}$ ), according to the following methods. Cells were isolated from the BM (bilateral femurs and tibias) and spleens of C57BL/6 donor mice in RPMI-1640 medium (MilliporeSigma) under sterile conditions, and red blood cells were lysed using ACK buffer (Gibco). T cells were then depleted from the $\mathrm{BM}$ by incubation for 60 minutes at $4^{\circ} \mathrm{C}$ with purified anti-Thy 1.2 antibody $\left(1 \mu \mathrm{g}\right.$ per $10^{7}$ cells, clone 30-H12, no azide/low endotoxin, BD Biosciences), followed by incubation for 60 minutes at $37^{\circ} \mathrm{C}$ in Cytotoxicity Medium (Cedarlane) containing a 1:10 dilution of LowTox-M Rabbit Complement (Cedarlane) according to the manufacturer's instructions. T cell-depleted BM cells and splenocytes were washed, filtered, and maintained in RPMI-1640 medium prior to injection. T cell-depleted BM cells alone $\left(1 \times 10^{7}\right.$ per mouse in $200 \mu \mathrm{l}$ volume $)$ or T cell-depleted BM cells plus splenocytes $\left(1 \times 10^{7}\right.$ plus $1 \times 10^{6}$, respectively, per mouse in $200 \mu 1$ volume) were injected via lateral tail vein using a sterile syringe with a 31-gauge needle. Mice were housed in a specific pathogen-free barrier facility at Duke University.

ENTO (GS-9973) administration. On day +12 following HCT, recipient mice were started on Purina Rodent Chow 5001 formulated with ENTO at a concentration of $0.06 \%$ or $0.02 \%$ or with placebo (Research Diets Inc.). Each chow formulation also contained an inert dye introduced by the manufacturer for identification purposes. During the first week of administration, chow in each treatment group was weighed prior to feeding and after 1, 3, and 6 days, with the average chow consumption per mouse calculated at each time point.

Blood sampling. To obtain plasma for pharmacokinetic studies and blood cells for flow cytometry analysis, mice were bled from the orbital sinus using a sterile syringe with 31-gauge needle for a total maximum volume of $50 \mu \mathrm{l}$ per blood draw, and the blood was transferred immediately to heparinized tubes. The same mouse was never bled more than once within any 1-week period. Plasma was isolated by an initial centrifugation step at $300 \mathrm{~g}$ for 5 minutes to remove cells, followed by a second centrifugation step at $8,000 \mathrm{~g}$ for 5 minutes to remove any remaining insoluble debris. Plasma samples were stored at $-80^{\circ} \mathrm{C}$ until used for pharmacodynamic studies.

Flow cytometry analysis. To determine blood cell frequencies and numbers, an equivalent volume of whole blood from each mouse was washed in DPBS and the red blood cells were lysed with ACK buffer (Gibco) for 10 minutes at room temperature. The cells were then washed with DPBS twice, followed by initial staining using a Zombie Aqua Fixable Viability Kit (BioLegend) to detect dead cells, along with TruStain fcX (BioLegend) to block Fc receptor binding of antibodies. The cells were then stained with a combination of fluorochrome-conjugated antibodies against mouse CD19 (clone 6D5, BioLegend), CD3 (clone 17A2, BioLegend), $\mathrm{H}-2 \mathrm{~Kb}$ (clone AF6-88.5, BioLegend), and H-2Kd (clone SF1-1.1, BioLegend) for 30 minutes on ice in DPBS containing 2\% FBS. The cells were washed and resuspended in DPBS containing 2\% FBS along with a fixed volume of CountBright Absolute Counting Beads (Thermo Fisher Scientific) to enable cell enumeration during analysis. In some experiments, 7-AAD (BD Biosciences) was also used as a cell viability dye to detect dead cells. Cells were analyzed on a 3-laser BD FACSCanto flow cytometer (BD Immunocytometry Systems) followed by further analysis using the FlowJo vX software package. B cells and T cells were identified by CD19 and CD3 expression, respectively, for cells with the forward scatter (FSC) and side scatter (SSC) properties of lymphocytes. Monocytes were identified by their distinct FSC and SSC properties along with additional negative staining for lymphocyte markers.

To assess spleen cell subsets in unstimulated cells, viably frozen splenocytes were thawed and washed with DPBS, followed by initial staining using a Zombie Aqua Fixable Viability Kit (BioLegend) 
to detect dead cells, along with TruStain fcX (BioLegend) to block Fc receptor binding of antibodies. To assess activated B cells, Tfh cells, and MDSCs, splenocytes were then stained with a combination of fluorochrome-conjugated antibodies against mouse B220 (clone RA3-6B2, BioLegend), GL7 (clone GL7, BioLegend), CD11b (clone M1/70, BD Biosciences), Ly6-C (clone HK1.4, BioLegend), Ly6-G (clone RB6-8C5, Thermo Fisher Scientific), CD4 (clone RM4-5, BD Biosciences), and CXCR5 (clone L138D7, BioLegend). To assess Tregs, splenocytes were surface stained using fluorochrome-conjugated CD4 antibody (clone GK1.5, BioLegend), fixed and permeabilized using an eBioscience Foxp3/Transcription Factor Staining Buffer Set (Thermo Fisher Scientific) according to the manufacturer's instructions, and then stained intracellularly using a fluorochrome-conjugated FoxP3 antibody (clone MF-14, BioLegend). To detect intracellular cytokines for assessing Th1, Th2, and Th17 cells, splenocytes were cultured for 5 hours in the presence of $50 \mathrm{ng} / \mathrm{ml} \mathrm{PMA}$ and $1 \mu \mathrm{g} / \mathrm{ml}$ ionomycin in RPMI-1640 medium supplemented with 10\% FBS, $55 \mu \mathrm{M}$ 2-mercaptoethanol, and BD GolgiStop Protein Transport Inhibitor (BD Biosciences) at the manufacturer's recommended dilution. The cells were then harvested and washed in DPBS, followed by initial staining using a Zombie Aqua Fixable Viability Kit (BioLegend) to detect dead cells, along with TruStain fcX (BioLegend) to block Fc receptor binding of antibodies. The cells were then fixed, permeabilized, and stained using a Mouse Th1/Th2/Th17 Phenotyping Kit (catalog 560758, BD Biosciences) according to the manufacturer's instructions. All above cell samples were analyzed on a 3-laser BD FACSCanto flow cytometer, followed by further analysis using the FlowJo vX software package.

To assess apoptosis of human B cells, T cells, and monocytes, PBMCs were cultured for 48 hours in RPMI-1640 medium containing 10\% FBS and $55 \mu \mathrm{M}$ 2-mercaptoethanol, along with various concentrations of ENTO, and then harvested, pelleted, and resuspended in wash buffer (PBS containing 2\% FBS) containing Fc block (Human TruStain FcX, BioLegend) at the concentration recommended by the manufacturer. Following a 15-minute incubation on ice, the cells were stained with fluorochrome-conjugated antibodies against human CD19 (clone HIB19, BioLegend), CD3 (clone SK7, BioLegend), and CD14 (clone RMO52, Beckman-Coulter) for an additional 30 minutes. The cells were then washed in cold PBS, followed by a second wash with Annexin V Binding Buffer (Annexin V Apoptosis Detection Kit, eBioscience) according to the manufacturer's instructions. The cells were then resuspended in Annexin V Binding Buffer containing fluorochrome-conjugated Annexin $\mathrm{V}$ and incubated in the dark for 15 minutes at room temperature. Finally, the cells were washed with cold Annexin V Binding Buffer, resuspended in cold Annexin V Binding Buffer containing 7-AAD (BD Biosciences), kept on ice, and analyzed immediately on a FACSCanto flow cytometer. Flow cytometry data files were analyzed using FlowJo vX software to identify subsets and determine the frequencies of apoptotic cells based on Annexin V and 7-AAD staining.

Clinical eye score assessment. Mice were evaluated for various clinical manifestations of eye pathology by an expert masked investigator from the Duke Eye Center specializing in models of eye disease in the mouse. Various eye disease criteria were assessed by biomicroscopy, as described previously (42), and were scored using a validated, graded scoring system as follows and as described in the legend for Figure 2. For chemosis, eyelid edema, conjunctival redness, and tearing, scores for each eye ranged from 0 (not detectable) to 3.0 (most severe) as follows: 0, none; $0.25-1.0$, mild; $1.25-2.0$, moderate; and 2.25-3.0, severe. Blepharitis and mucoid discharge were scored as either being absent (scored as 0 ) or present (scored as 1). A clinical score for each criteria in an individual mouse was then determined by summing the masked scores for both eyes (right plus left).

Clinical skin score assessment. Mice were evaluated for various clinical manifestations of skin GVHD by an expert masked investigator from the Duke Department of Pathology. Various skin disease criteria were scored as follows and as also described in the legend for Figure 8: dermis ( 0 , normal; 1 , mild increased collagen density; 2 , marked increased collagen density); hair follicles ( 0 , normal number of approximately 5 per linear millimeter; 1 , between 1 and 5 follicles per linear millimeter; $2,<1$ follicle per linear millimeter); epidermis ( 0 , normal; 1 , interface damage in $<20 \%$ of section with occasional necrotic keratinocytes; 2 , widespread interface damage [ $>20 \%$ of section]); and inflammation ( 0 , none; 1 , focal infiltrates; 2, widespread infiltrates).

Histochemistry and immunohistochemistry. For mice, tissues were procured following natural death or euthanasia and fixed in 4\% paraformaldehyde in PBS (Santa Cruz Biotechnology) and then processed as described below by the Duke Department of Pathology Research Histology Core on a fee-for-service basis. Paraffin-embedded sections $(5 \mu \mathrm{m})$ were prepared and then stained with $\mathrm{H} \& \mathrm{E}$ and Masson's trichrome 
according to the Research Histology Core's standard procedures. For detection of SYK, sections were stained with rabbit monoclonal Ab specific for mouse/human SYK (clone D3Z1E, Cell Signaling Technology) followed by colorimetric detection with peroxidase-conjugated anti-rabbit IgG Ab and counterstaining with hematoxylin, according to the Research Histology Core's standard procedures. For detection of F4/80, sections were stained with rat monoclonal Ab specific for mouse F4/80 (clone Cl:A3-1, Bio-Rad), followed by colorimetric detection with peroxidase-conjugated anti-rat IgG $\mathrm{Ab}$ and counterstaining with hematoxylin, according to the Research Histology Core's standard procedures.

For human cGVHD patients, FFPE biopsies from lichenoid skin lesions were sectioned (5 $\mu \mathrm{m})$ and then stained with rabbit monoclonal Ab specific for human/mouse SYK (clone D3Z1E, Cell Signaling Technology) or with mouse monoclonal Ab specific for human CD19 (clone CD19, Biocare Medical), followed by colorimetric detection with peroxidase-conjugated anti-rabbit or anti-mouse IgG Ab and counterstaining with hematoxylin, all according to the Research Histology Core's standard procedures.

Natural IgM antibody ELISA. ELISA plate wells were coated overnight at $4^{\circ} \mathrm{C}$ with $1 \times 10^{6} \mathrm{pfu} / \mathrm{ml} \mathrm{VV}$ (Western Reserve strain) in coating buffer $\left(0.1 \mathrm{M} \mathrm{NaHCO}_{3}, \mathrm{pH} 9.6\right)$. Wells were then washed 3 times with DPBS containing $0.05 \%$ Tween 20, blocked for 2 hours with DPBS containing $10 \%$ FBS, washed again 3 times with DPBS containing $0.05 \%$ Tween 20, and then incubated for 2 hours at room temperature with plasma samples diluted at 2-fold serial dilutions in DPBS containing $2 \%$ FBS. The wells were then washed 5 times with DPBS containing 0.05\% Tween 20 and incubated for 1 hour at room temperature with HRP-conjugated goat anti-mouse IgM secondary antibody (catalog 1020-05, SouthernBiotech). The wells were washed 5 times with DPBS containing $0.05 \%$ Tween 20 and then developed using a TMB substrate kit (Thermo Fisher Scientific) according to the manufacturer's instructions. The reaction was stopped with $2 \mathrm{M}$ sulfuric acid, and the absorbance was read at $450 \mathrm{~nm}$.

Statistics. Statistical significance among experimental groups was determined using 1-way ANOVA with Tukey's multiple comparisons test, log-rank test, or unpaired 2-tailed Student's $t$ test (all with GraphPad Prism, version 7), as detailed in the figure legends. $P$ values of less than 0.05 were considered significant.

Study approval. All procedures in mice were performed under a protocol approved by the Duke University Institutional Animal Care and Use Committee. Clinical sample protocols for patient blood and skin biopsy samples were approved by the Institutional Review Board at Duke University.

\section{Author contributions}

JCP, JADP, JYK, JSS, DRS, NJC, and SS designed the study; JCP, WJ, NJR, HS, ARC, and DMC performed the experiments, acquired the data, and analyzed the data; DRS, VLP, and BJC provided scientific advice and reviewed the paper; and JCP, JADP, JYK, and SS wrote the paper.

\section{Acknowledgments}

This work was supported by a grant from the National Institutes of Health (R01 HL 129061 [National Heart, Lung, and Blood Institute] to SS) and by a sponsored research agreement between Duke University and Gilead Sciences. We also thank Yiping Yang and Jiangao Zhu for providing reagents and protocols to perform VV ELISAs.

Address correspondence to: Stefanie Sarantopoulos, Duke Adult Bone Marrow, 2400 Pratt Street, Suite 9000, DUMC Box 3961, Durham, North Carolina 27710, USA. Phone: 919.668.4383; Email: stefanie.sarantopoulos@duke.edu.

NJR's present address is: Shire Pharmaceuticals, Seattle, Washington, USA.

\footnotetext{
1. Zeiser R, Blazar BR. Acute graft-versus-host disease - biologic process, prevention, and therapy. $N$ Engl J Med. 2017;377(22):2167-2179.

2. Zeiser R, Socié G, Blazar BR. Pathogenesis of acute graft-versus-host disease: from intestinal microbiota alterations to donor T cell activation. Br J Haematol. 2016;175(2):191-207.

3. Cutler CS, Koreth J, Ritz J. Mechanistic approaches for the prevention and treatment of chronic GVHD. Blood. 2017;129(1):22-29.

4. Lee SJ. Classification systems for chronic graft-versus-host disease. Blood. 2017;129(1):30-37.

5. Zeiser R, Blazar BR. Pathophysiology of chronic graft-versus-host disease and therapeutic targets. $N$ Engl J Med. 2017;377(26):2565-2579.

6. Zeiser R, Negrin RS. Introduction to a review series on chronic GVHD: from pathogenic B-cell receptor signaling to novel
} 
therapeutic targets. Blood. 2017;129(1):1-2.

7. Chen BJ, Cui X, Sempowski GD, Liu C, Chao NJ. Transfer of allogeneic CD62L- memory T cells without graft-versus-host disease. Blood. 2004;103(4):1534-1541.

8. Chen BJ, et al. Inability of memory T cells to induce graft-versus-host disease is a result of an abortive alloresponse. Blood. 2007;109(7):3115-3123.

9. MacDonald KP, Hill GR, Blazar BR. Chronic graft-versus-host disease: biological insights from preclinical and clinical studies Blood. 2017;129(1):13-21.

10. MacDonald KP, Shlomchik WD, Reddy P. Biology of graft-versus-host responses: recent insights. Biol Blood Marrow Transplant. 2013;19(1 Supp1):S10-S14.

11. Zhang P, Chen BJ, Chao NJ. Prevention of GVHD without losing GVL effect: windows of opportunity. Immunol Res. 2011;49(1-3):49-55.

12. Zhang P, Wu J, Deoliveira D, Chao NJ, Chen BJ. Allospecific CD4(+) effector memory T cells do not induce graft-versus-host disease in mice. Biol Blood Marrow Transplant. 2012;18(10):1488-1499.

13. Sullivan KM, et al. Chronic graft-versus-host disease in 52 patients: adverse natural course and successful treatment with combination immunosuppression. Blood. 1981;57(2):267-276.

14. Lee SJ, et al. Success of immunosuppressive treatments in patients with chronic graft-versus-host disease. Biol Blood Marrow Transplant. 2018;24(3):555-562.

15. Martin PJ, et al. An endpoint associated with clinical benefit after initial treatment of chronic graft-versus-host disease. Blood. 2017;130(3):360-367.

16. Chen BJ, Cui X, Liu C, Chao NJ. Prevention of graft-versus-host disease while preserving graft-versus-leukemia effect after selective depletion of host-reactive T cells by photodynamic cell purging process. Blood. 2002;99(9):3083-3088.

17. Socié G, Ritz J. Current issues in chronic graft-versus-host disease. Blood. 2014;124(3):374-384.

18. Soiffer RJ, et al. Prospective, randomized, double-blind, phase III clinical trial of anti-T-lymphocyte globulin to assess impact on chronic graft-versus-host disease-free survival in patients undergoing HLA-matched unrelated myeloablative hematopoietic cell transplantation. J Clin Oncol. 2017;35(36):4003-4011.

19. Jin $\mathrm{H}$, et al. Antibodies from donor B cells perpetuate cutaneous chronic graft-versus-host disease in mice. Blood. 2016;127(18):2249-2260.

20. Srinivasan $\mathrm{M}$, et al. Donor B-cell alloantibody deposition and germinal center formation are required for the development of murine chronic GVHD and bronchiolitis obliterans. Blood. 2012;119(6):1570-1580.

21. Zhang C, et al. Donor CD4+ T and B cells in transplants induce chronic graft-versus-host disease with autoimmune manifestations. Blood. 2006;107(7):2993-3001.

22. Cutler C, et al. Rituximab prophylaxis prevents corticosteroid-requiring chronic GVHD after allogeneic peripheral blood stem cell transplantation: results of a phase 2 trial. Blood. 2013;122(8):1510-1517.

23. Sarantopoulos S, et al. Recovery of B-cell homeostasis after rituximab in chronic graft-versus-host disease. Blood. 2011;117(7):2275-2283.

24. Sarantopoulos S, Blazar BR, Cutler C, Ritz J. B cells in chronic graft-versus-host disease. Biol Blood Marrow Transplant. 2015;21(1):16-23.

25. Sarantopoulos S, et al. High levels of B-cell activating factor in patients with active chronic graft-versus-host disease. Clin Cancer Res. 2007;13(20):6107-6114.

26. Sarantopoulos S, et al. Altered B-cell homeostasis and excess BAFF in human chronic graft-versus-host disease. Blood. 2009;113(16):3865-3874.

27. Mócsai A, Ruland J, Tybulewicz VL. The SYK tyrosine kinase: a crucial player in diverse biological functions. Nat Rev Immunol 2010;10(6):387-402.

28. Flynn R, et al. Targeting Syk-activated B cells in murine and human chronic graft-versus-host disease. Blood. 2015;125(26):4085-4094.

29. Allen JL, et al. B cells from patients with chronic GVHD are activated and primed for survival via BAFF-mediated pathways. Blood. 2012;120(12):2529-2536.

30. Allen JL, et al. Increased BCR responsiveness in B cells from patients with chronic GVHD. Blood. 2014;123(13):2108-2115.

31. Zeiser R, Sarantopoulos S, Blazar BR. B-cell targeting in chronic graft-versus-host disease. Blood. 2018;131(13):1399-1405.

32. Sarantopoulos S, Ritz J. Aberrant B-cell homeostasis in chronic GVHD. Blood. 2015;125(11):1703-1707.

33. Le Huu D, et al. Blockade of Syk ameliorates the development of murine sclerodermatous chronic graft-versus-host disease. J Dermatol Sci. 2014;74(3):214-221.

34. Im A, Hakim FT, Pavletic SZ. Novel targets in the treatment of chronic graft-versus-host disease. Leukemia. 2017;31(3):543-554.

35. Leonhardt F, et al. Spleen tyrosine kinase (Syk) is a potent target for GvHD prevention at different cellular levels. Leukemia. 2012;26(7):1617-1629

36. Geahlen RL. Getting Syk: spleen tyrosine kinase as a therapeutic target. Trends Pharmacol Sci. 2014;35(8):414-422

37. Tohyama Y, Yamamura H. Protein tyrosine kinase, syk: a key player in phagocytic cells. J Biochem. 2009;145(3):267-273.

38. Alexander KA, et al. CSF-1-dependant donor-derived macrophages mediate chronic graft-versus-host disease. J Clin Invest. 2014;124(10):4266-4280.

39. Burke RT, et al. A potential therapeutic strategy for chronic lymphocytic leukemia by combining Idelalisib and GS-9973, a novel spleen tyrosine kinase (Syk) inhibitor. Oncotarget. 2014;5(4):908-915.

40. Clemens GR, et al. Developmental toxicity associated with receptor tyrosine kinase Ret inhibition in reproductive toxicity testing. Birth Defects Res Part A Clin Mol Teratol. 2009;85(2):130-136.

41. Currie KS, et al. Discovery of GS-9973, a selective and orally efficacious inhibitor of spleen tyrosine kinase. JMed Chem. 2014;57(9):3856-3873.

42. Magone MT, Chan CC, Rizzo LV, Kozhich AT, Whitcup SM. A novel murine model of allergic conjunctivitis. Clin Immunol Immunopathol. 1998;87(1):75-84.

43. Schlereth S, Lee HS, Khandelwal P, Saban DR. Blocking CCR7 at the ocular surface impairs the pathogenic contribution of dendritic cells in allergic conjunctivitis. Am J Pathol. 2012;180(6):2351-2360. 
44. Di Ianni M, et al. Tregs prevent GVHD and promote immune reconstitution in HLA-haploidentical transplantation. Blood. 2011;117(14):3921-3928.

45. Koreth J, et al. Interleukin-2 and regulatory T cells in graft-versus-host disease. N Engl J Med. 2011;365(22):2055-2066.

46. Matsuoka K, et al. Altered regulatory T cell homeostasis in patients with CD4+ lymphopenia following allogeneic hematopoietic stem cell transplantation. J Clin Invest. 2010;120(5):1479-1493.

47. Matsuoka K, et al. Low-dose interleukin-2 therapy restores regulatory $\mathrm{T}$ cell homeostasis in patients with chronic graft-versushost disease. Sci Transl Med. 2013;5(179):179ra43.

48. Young JS, et al. Donor B cells in transplants augment clonal expansion and survival of pathogenic CD4+ T cells that mediate autoimmune-like chronic graft-versus-host disease. J Immunol. 2012;189(1):222-233.

49. Davies HA, Clark JD, Dalton KJ, Edwards OM. Insulin requirements of diabetic women who breast feed. $B M J$. 1989;298(6684):1357-1358.

50. Flynn R, et al. Increased T follicular helper cells and germinal center B cells are required for cGVHD and bronchiolitis obliterans. Blood. 2014;123(25):3988-3998.

51. Wu T, et al. Thymic damage, impaired negative selection, and development of chronic graft-versus-host disease caused by donor CD4+ and CD8+ T cells. J Immunol. 2013;191(1):488-499.

52. Poe JC, et al. An aberrant NOTCH2-BCR signaling axis in B cells from patients with chronic GVHD. Blood. 2017;130(19):2131-2145.

53. Gowdy KM, et al. Protective role of T-bet and Th1 cytokines in pulmonary graft-versus-host disease and peribronchiolar fibrosis. Am J Respir Cell Mol Biol. 2012;46(2):249-256.

54. Nikolic B, Lee S, Bronson RT, Grusby MJ, Sykes M. Th1 and Th2 mediate acute graft-versus-host disease, each with distinct end-organ targets. J Clin Invest. 2000;105(9):1289-1298.

55. Yi T, et al. Reciprocal differentiation and tissue-specific pathogenesis of Th1, Th2, and Th17 cells in graft-versus-host disease. Blood. 2009;114(14):3101-3112.

56. Coghill JM, Sarantopoulos S, Moran TP, Murphy WJ, Blazar BR, Serody JS. Effector CD4+ T cells, the cytokines they generate, and GVHD: something old and something new. Blood. 2011;117(12):3268-3276.

57. Zhao D, et al. In vivo-activated CD103+CD4+ regulatory T cells ameliorate ongoing chronic graft-versus-host disease. Blood. 2008;112(5):2129-2138.

58. Alho AC, et al. Unbalanced recovery of regulatory and effector T cells after allogeneic stem cell transplantation contributes to chronic GVHD. Blood. 2016;127(5):646-657.

59. Beres AJ, Drobyski WR. The role of regulatory T cells in the biology of graft versus host disease. Front Immunol. 2013;4:163

60. Edinger M, et al. CD4+CD25+ regulatory T cells preserve graft-versus-tumor activity while inhibiting graft-versus-host disease after bone marrow transplantation. Nat Med. 2003;9(9):1144-1150.

61. Mutis T, et al. Human regulatory T cells control xenogeneic graft-versus-host disease induced by autologous T cells in RAG2-/gammac-/- immunodeficient mice. Clin Cancer Res. 2006;12(18):5520-5525.

62. Zorn E, et al. Reduced frequency of FOXP3+ CD4+CD25+ regulatory T cells in patients with chronic graft-versus-host disease. Blood. 2005;106(8):2903-2911

63. Bronte V, et al. Recommendations for myeloid-derived suppressor cell nomenclature and characterization standards. Nat Commun. 2016;7:12150

64. Ochsenbein AF, et al. Control of early viral and bacterial distribution and disease by natural antibodies. Science. 1999;286(5447):2156-2159.

65. Sharman J, et al. An open-label phase 2 trial of entospletinib (GS-9973), a selective spleen tyrosine kinase inhibitor, in chronic lymphocytic leukemia. Blood. 2015;125(15):2336-2343.

66. Ramanathan S, et al. Pharmacokinetics, pharmacodynamics, and safety of entospletinib, a novel pSYK inhibitor, following single and multiple oral dosing in healthy volunteers. Clin Drug Investig. 2017;37(2):195-205

67. de Masson A, et al. CD24(hi)CD27 ${ }^{+}$and plasmablast-like regulatory B cells in human chronic graft-versus-host disease. Blood 2015;125(11):1830-1839.

68. Taylor PA, Lees CJ, Blazar BR. The infusion of ex vivo activated and expanded CD4(+)CD25(+) immune regulatory cells inhibits graft-versus-host disease lethality. Blood. 2002;99(10):3493-3499.

69. Koreth J, et al. Efficacy, durability, and response predictors of low-dose interleukin-2 therapy for chronic graft-versus-host disease. Blood. 2016;128(1):130-137. 